Revue d'histoire de l'Amérique française

ZRS REVUE D.HISTOIRE DE L'AMÉRIQUE FRANÇAISE

\title{
La liste civile du Bas-Canada (1794-1812) : Un essai d'économie historique (suite et fin)
}

\section{Gilles Paquet et Jean-Pierre Wallot}

Volume 24, numéro 2, septembre 1970

URI : https://id.erudit.org/iderudit/302975ar

DOI : https://doi.org/10.7202/302975ar

Aller au sommaire du numéro

Éditeur(s)

Institut d'histoire de l'Amérique française

ISSN

0035-2357 (imprimé)

1492-1383 (numérique)

Découvrir la revue

Citer cet article

Paquet, G. \& Wallot, J.-P. (1970). La liste civile du Bas-Canada (1794-1812) : Un essai d'économie historique (suite et fin). Revue d'histoire de l'Amérique française, 24(2), 251-286. https://doi.org/10.7202/302975ar d'utilisation que vous pouvez consulter en ligne. 


\title{
LA LISTE CIVILE DU BAS-CANADA (1794-1812) : UN ESSAI D'ÉCONOMIE HISTORIQUE*
}

\author{
(suite et fin)
}

\section{La liste civile : microcosme d'une situation globale}

Entre 1794 et 1812 , la liste civile évolue au rythme de la vie bas-canadienne: elle suit un sentier de croissance largement parallèle à l'expansion démographique; elle réagit dans sa structure aux besoins nouveaux et plus différenciés issus de la restructuration des échanges internationaux; elle est enfin un lieu privilégié d'arbitrage des heurts plus ou moins violents entre groupes et factions du Bas-Canada. ${ }^{866}$ Plus encore, non seulement enregistre-t-elle les tiraillements de ces pulsions diverses; elle incorpore aussi les biais qu'injecte l'Etat dans sa perception des besoins collectifs et dans ses opérations. ${ }^{187}$

Dans les sections précédentes, nous avons disséqué ces diverses forces opérant autour et à l'intérieur de la liste civile comme institution. L'effort de conceptualisation, la chronique des conflits et les analyses empiriques ont décelé une série de mécanismes qui sous-tendent le fonctionnement de l'économie publique bas-canadienne. La présente section entend faire le joint avec la situation globale de la société bas-canadienne au tournant du $\mathrm{XIX}^{\mathrm{e}}$ siècle et montrer que la liste civile en a été en quelque sorte un microcosme.

La clé de ce passage au niveau global, nous la chercherons dans un élargissement du concept de patronage, selon un procédé que nous avons amorcé à la note 56. Dans le Bas-Canada, disionsnous, le patronage embrasse les plans politique, social, familial, etc. Il nous faut ordonner cet enchevêtrement et tenter de rejoindre, par-delà nos propos sur les pensions et les salaires, la polyvalence des affrontements entre groupes au nom d'intérêts plus ou moins clairs et de motivations plus ou moins avouées.

*Voir notre Revue, 23 (1969-1970) : 209-230, 361-392; 24 (1970-1971) : 3-43. Nous remercions vivement nos attachées de recherche, Françoise Faribault, Jane McCraw et Rita Wallot. 186 Groupes et factions qui perçoivent la réalité de façon souvent contradictoire (v.g. voir début section V). 187 Voir notre Revue, 23 (1969-1970) : 217-219, 373-374. 


\section{Le patronage comme indicateur social}

Si le patronage, comme l'affirme Boissevain, s'avère ce nœud complexe de relations entre, d'une part, ceux qui usent de leur influence, de leur position sociale ou de quelque attribut pour en protéger d'autres, et, par ailleurs, ceux qui bénéficient de cette aide, ${ }^{188}$ on peut donc analyser la nature du patronage en termes des faveurs échangées, des partenaires de ce "commerce" et des motivations sous-jacentes. Ces composantes, à leur tour, constituent un indicateur utile du type de société à l'étude. Il y aura toujours de telles relations; mais selon la structure sociale d'une société donnée, les motivations des groupes, la nature et le sens des faveurs échangées varieront.

Dans le cas d'une société en voie de transformation rapide, le recours au patronage peut être un instrument fort important pour faciliter les ajustements dans un univers où trop souvent les institutions nécessaires ne surgissent qu'après de longs délais. En ces périodes de transition, il faut s'attendre à la coexistence de rationalités et de notions d'efficacité diverses. Ces ambiguïtés devraient se refléter dans la structure du patronage: tributaire des sociétés en voie de disparition et d'apparition, ce dernier doit se constituer en une espèce hybride justiciable des deux cadres de référence.

Pour fixer les idées, posons un continuum entre deux pôles définis par le patronage paternaliste ("patron-client patronage") qu'ont étudié surtout les anthropologues, ${ }^{189}$ et le patronage politique, qui intéresse d'abord les politicologues. ${ }^{190}$ Le premier, plus archaïque dans ses formes, se réfère à un type de relation sociale; le second, plus moderne, à un aspect du fonctionnement de l'Etat. A mesure qu'une société se modernise, s'opère la transition depuis une dominante paternaliste à une dominante politique. ${ }^{191}$

Dans toute société donnée, ces deux formes de patronage coexistent. La composante paternaliste prédomine dans des so-

188 Voir note 56.

189 Les anthropologues s'intéressent au "kinship behavior", "[to] the analysis of how persons of unequal authority, yet linked through ties of interest and friendship, manipulate their relationships in order to attain their ends" (A. Weingrod, "Patrons, Patronage, and Political Parties", 377, 380 ).

190 "This . . patronage refers to the ways in which party politicians distribute public jobs or special favors in exchange for electoral support." (Ibid., 380).

191 S. Silverman, cité dans ibid., 382. 
ciétés segmentées, où l'Etat se contente de maintenir l'ordre. $\mathrm{Au}$ contraire, la composante politique s'amplifie à mesure que s'accroît le pouvoir de l'Etat et que se fait mieux sentir son interventionnisme. On peut donc lier la montée de la composante politique à la plus grande activité de l'Etat tant dans l'allocation que dans la distribution des ressources. ${ }^{192}$ Et nous devrions alors discerner, au niveau de la liste civile, un glissement dans la fonction de médiation du patronage qui accompagne les restructurations de la société canadienne au tournant du XIX ${ }^{\mathrm{e}}$ siècle.

Le patronage paternaliste, dont le type pur s'incarnerait dans une société féodale d'Ancien Régime, n'a peut-être jamais joué entièrement au Canada, à cause de l'indépendance des habitants ainsi que des conditions géographiques, économiques et sociales dans la colonie. Toutefois, comme nous le verrons, les seigneurs, au tournant du $\mathrm{XIX}^{\mathrm{e}}$ siècle, conçoivent le patronage selon un schème paternaliste. D'autre part, avec la restructuration de l'économie, l'extension considérable des activités de l'Etat et la mise en place de nouvelles règles du jeu politique (une Assemblée législative et élective, en 1791), le patronage politique prend de plus en plus d'importance. De distortions à d'autres niveaux (freins coloniaux à l'autonomie administrative de l'Etat, opposition des groupes sociaux et ethniques), il s'ensuit que ce sont surtout les bureaucrates qui jouissent du patronage et peuvent l'employer pour influencer l'électorat. Le parti canadien, majorité à l'Assemblée, combat farouchement pour s'en approprier le contrôle. Bref, à côté d'un groupe en perte de vitesse (les seigneurs) qui s'incruste dans une forme archaïque de patronage, il semble bien que la rivalité entre le parti britannique ou gouvernemental et le parti canadien pour la mainmise sur le patronage, s'assimile avant tout à une lutte pour le pouvoir politique et, au-delà, pour l'autorité sur la société globale.

Tel est en tout cas le sens de l'hypothèse que nous développerons dans cette section. Les transformations de l'économie

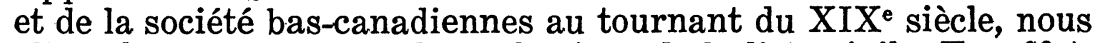
allons les retracer dans les pulsations de la liste civile. En effet, ce mélange curieux de patronage paternaliste et de patronage

192 D'un point de vue structurel, "patron-client ties can be seen to arise within a state structure in which authority is dispersed and state activity limited in scope, and in which considerable separation exists between the levels of village, city and state. Party-directed patronage, on the other hand, is associated with the expanding scope and general proliferation of state activities, and also with the growing integration of village, city and state ... The 'politics of development' is thus a central cause of the expansion of party patronage." (A. Weingrod, ibid., 381, 384). 
politique est un indicateur précieux, et son analyse nous permettra de saisir certaines des tendances de toute la société bascanadienne à cette époque. Ce procédé cherchera dans la dynamique de la liste civile le profil de la dynamique de la société canadienne dans laquelle elle s'enracine.

\section{Quelques vues un peu courtes}

Avant de franchir ce passage à la société globale dont il a été question à la section I, il nous a paru important de rappeler quelques vues un peu courtes sur les querelles autour de la liste civile. Deux en particulier, bien qu'à des degrés divers, nous semblent mériter une attention spéciale: l'une voudrait conférer à ces affrontements une dimension presque exclusivement constitutionnelle; l'autre les considère comme un combat autour du pot au beurre. L'une et l'autre vues simplifient à outrance une réalité bien plus complexe.

a) De façon générale, l'historingraphie a interprété les conflits autour de la liste civile et des subsides en fonction de problèmes surtout constitutionnels. ${ }^{193}$ Mais la lutte politique, importante sans doute, sert d'arène à plusieurs équipes. Celles-ci s'agrègent autour des intérêts les plus divers qui touchent à tous les aspects de la vie. Les motivations derrière ces crispations politiques débordent les cadres strictement constitutionnels. Les équipes politiques en présence représentent des groupes sociaux, ethniques et régionaux. De plus, comme nous l'avons vu dans les sections V et VI, l'Etat n'est pas neutre. Sa lecture de la situation et ses opérations trahissent des préférences sociales, ethniques et peut-être même régionales. Ainsi, la minorité britannique accapare la majorité des places à tous les paliers, même si cette suprématie, sur le plan de la répartition, diminue légèrement avec le temps. ${ }^{194} \mathrm{En}$ outre, la noblesse seigneuriale et militaire s'approprie une proportion considérable du petit nombre de places disponibles pour les Canadiens - nombre encore restreint par les cumuls, environ le quart des emplois après 1800 : les nobles raflent environ la moitié des places et des pen-

193 Voir note 159.

${ }_{194} \mathrm{Au}$ niveau du patronage de rétribution, l'avantage en faveur des Britanniques s'accroît avec le temps, légèrement dans le cas des salaires, de façon radicale dans le cas des pensions. Mais au niveau du patronage de répartition, les chances des Canadiens français d'obtenir une place croissent avec le temps, même s'ils ne dépassent jamais 40 pour cent du total. Bien entendu, leur proportion dans l'ensemble de la population et leur croissance démographique sont sans commune mesure avec cette amélioration relative. Voir la section VI. 
sions, même en fin de période, et la quasi-totalité de celles au-dessus de $£ 100$ st. La part des emplois allant aux professionnels demeure très restreinte et stable: là encore, ce sont surtout des nobles ou des partisans du gouvernement à l'Assemblée qui les décrochent.

Ce faisceau de groupes comprend des entités à valences politiques bien différentes. On ne saurait réduire leurs activités disparates à un commun dénominateur aussi abstrait que la "lutte constitutionnelle". Aussi peut-on penser que les affrontements entre ces groupes sociaux divers sont ancrés dans des motivations plus matérielles. C'est là l'option qu'a faite l'historiographie moderne.

b) En effet, certains travaux récents ont tendance à ramener ces tensions (qu'enregistre la liste civile, mais qui sont le fait de la société ainsi que nous voudrons le montrer) à une source majeure: les intérêts mesquins d'un groupe particulier, les professionnels. ${ }^{195}$ De fait, la réalité nous semble plus riche et plus complexe: elle résiste à cette sorte de réduction sociale tout autant qu'à la réduction constitutionnelle. La liste civile ouvre sur l'ensemble de la société bas-canadienne, sur le large éventail des groupes et de leurs motivations, sur leurs confrontations au niveau de la praxis et de la maîtrise de l'Etat. ${ }^{196}$

Les leaders du parti canadien mènent la lutte pour que l'Assemblée élue contrôle les subsides. Pour la plupart, ce sont des professionnels, surtout des avocats et des notaires. Ils invoquent avant tout des arguments constitutionnels et ethniques (voir section IV). Mais on peut supposer que certains d'entre eux aspirent à des emplois publics et, éventuellement, à une pension. Leur doctrine constitutionnelle, à ce moment, prévoit d'ailleurs et avalise une présence majoritaire des Canadiens dans les différents services du gouvernement. ${ }^{197}$ Mais la fonction

195 V.g. F. Ouellet, op. cit., chapitre VII, surtout p. 203 ; W. L. Morton, The Kingdom of Canada (Toronto, 1963), 218; aussi note 162.

196 Il s'agit d'un bel exemple d" "économie généralisée". Pour un exposé de notre modèle général, voir G. Paquet et J.-P. Wallot, "Canada 17601850 : anamorphoses et prospective", in Economie québécoise (cahiers 1-2 de l'Úniversité du Québec, novembre 1969), 282-289.

197 Il en ira différemment dans les années 1820 et 1830 . Pierre Bédard, le chef du parti canadien, accepte une place de juge à Trois-Rivières, en 1812, sans que l'on songe à le lui reprocher. Bédard, un avocat fort occupé, se débat parfois dans des difficultés financières; mais celles-ci tiennent à ses problèmes domestiques. D'autre part, il semble bien qu'il espère une promotion depuis Trois-Rivières au banc de Montréal ou de Québec, ce qui lui assurerait presque automatiquement un siège au Conseil exécutif et 
publique ne peut accommoder qu'une proportion infime de chefs canadiens, à cause du nombre très restreint d'emplois "professionnels" - la stabilité du nombre de fonctionnaires professionnels, même dans le cas des privilégiés (nobles, Britanniques), est éloquente à cet égard: ceux de juges, juristes de la Couronne (avocat général, solliciteur général), arpenteur général, greffier du papier terrier, juge de police (de 1810 à 1812). Encore ces emplois conviennent-ils presque tous à des avocats, non à des notaires qui forment pourtant le plus fort contingent de professionnels dans la province. ${ }^{198}$ Il faut leur ajouter le Conseil exécutif, qui compte généralement huit ou neuf membres: les émoluments ( $£ 100$ st. par année), intéressants parce qu'ils s'additionnent aux revenus ordinaires, paraissent toutefois secondaires à côté du pouvoir politique important que revêt un tel poste. Mais en gros, dans la fonction publique, il y a peu d'ouvertures intéressantes et accessibles pour les professionnels, particulièrement pour les notaires. On peut donc croire que si les professionnels convoitent ces postes rares, compte tenu d'une offre aüssi restreinte, ils devraient succomber facilement à la tentation d'emprunter une filière plus rapide et plus sûre: le changement d'allégeance. De "patriote", on devient "gouvernemental" (v.g. de Bonne, P. Panet). Or, l'ensemble du contingent, par le truchement du parti canadien, demeure rivé à des exigences fondamentales et au combat politique durant cette période. ${ }^{199}$

A la rigueur, une croissance anormale des professions libérales, comme on l'a suggéré, ${ }^{200}$ et des difficultés économiques, ${ }^{201}$

un salaire plus élevé. De là son intérêt constant pour la scène politique et son aigreur croissante par suite de la prolongation de ce qui devient un "exil". Avec le temps, ce qui a semblé un point de départ prometteur, sur tous les plans, devient un cul-de-sac. Voir les papiers Bédard aux Archives du Séminaire de Québec et dans les papiers Neilson, à Ottawa (APC, M.G. 24, B. 1, vol. 1, 2 et 31 ).

198 Par exemple, en 1812, la province compte 132 notaires (dont 10 Britanniques) comparativement à 51 avocats (dont 20 Britanniques) (Almanach de Québec [...] 1812 (Québec, 1812), 26-30). Voir aussi F. Ouellet, op. cit., 602 .

199 V.g. Bédard qui, même juge, collabore activement à la grande pétition au prince régent (1814).

200 Voir notes 162 et 195.

201 Un grand nombre d'actes notariés ont trait à des questions économiques : ventes, échanges, quittances, rentes, etc. Il est plausible qu'en période de récession, par exemple après la guerre de 1812, la baisse des affaires se répercute sérieusement sur le niveau de vie des notaires (comme aussi sur celui des avocats, bien que nous ne disposions pas, dans leur cas, d'un instrument de mesure aussi précis que dans celui des notaires). 
pourraient inciter les professionnels à se rabattre sur des postes subalternes mais sûrs, garantie au moins d'une survie modeste. Or, cette seconde possibilité ne colle pas très bien, elle non plus, à la réalité bas-canadienne d'avant 1812. En effet, malgré le grossissement des effectifs, ${ }^{202}$ les notaires et les avocats semblent se débrouiller très bien. Ils ne sont guère attirés par des postes dont la rémunération est somme toute relativement faible par rapport à ce que rapporte la pratique. Un examen des greffes de 50 notaires, sur un total de 178 qui ont pratiqué dans le Bas-Canada entre 1792 et 1812, révèle qu'un notaire préparait en moyenne 183 actes par année. Au nombre brut d'actes notariés, il faut ajouter les copies ou "expéditions" pour les différentes parties (quatre en moyenne par acte) au moment de la signature de l'acte et, au fil des ans, pour les actes accumulés dans le greffe, selon les besoins. Enfin, les lois canadiennes prévoient la présence obligatoire d'un second notaire en diverses circonstances (v.g. lors des inventaires après décès, de contestations de l'authenticité de certains documents). Or, dès 1789 , on s'insurge contre le niveau trop élevé et l'escalade constante des honoraires des notaires. ${ }^{203}$ Ces tarifs fluctuent d'un acte à l'autre, selon la nature, la complexité et la longueur du document. ${ }^{204}$

202 Pour une étude plus fouillée des revenus des notaires, qui tienne compte du temps et de la croissance des effectifs, voir G. Paquet et J.-P. Wallot, "La restructuration de l'économie et de la société canadienne au tournant du XIXe siècle", communication présentée au Ve Congrès International d'Histoire Economique, à Leningrad, août 1970. En gros, la moyenne annuelle de contrats pour un notaire semble s'élever de façon marquée entre 1796 et 1806 , puis tend à une baisse plus douce de 1807 à 1811 .

203 “... les Mrs civils de ce pays augmentent leurs honoraires de jour en jour, car ils lui ont pris 4 louis pour la copie de son acte de mariage, et 10 louis pour le partage, et pour le mien avec Mr Cugnet, 40." (A. Juchereau Duchesnay au comte des Méloizes, Beauport, 23 août 1789, APQ, lettres au comte des Méloizes). En 1808, un commentateur se moque des avocats et des notaires : le projet de loi à l'étude à l'Assemblée (en vue de réglementer le notariat - le projet de loi disparaît au Conseil législatif de sorte que nous n'en connaissons pas les dispositions) forcera les notaires à ne rédiger que de "bons actes ... suivant la loi". Or "il n'y a que les mauvais actes, qui grâce à Dieu sont très communs en ce moment, qui puissent nous [les avocats] faire vivre, donc si on nous en prive, nous sommes tous ruinés ... Trois donations mal faites m'ont valu plus de $£ 100$. Les testaments ! Quelle mine !" (Le Courier de Québec, 13 février 1808). Qu'à cela ne tienne, lui rétorque un autre correspondant, les arpenteurs sont si malhonnêtes ou ignorants qu'ils continueront à assurer une multiplicité de procès (ibid., 20 février 1808 ).

204 Une ordonnance de 1780 fixe dans le détail les honoraires des avocats et des notaires. Cependant, les taux semblent avoir grimpé considérablement à l'époque qui nous concerne. En plus, l'ordonnance prévoit que pour tout acte avec des clauses particulières, un notaire peut réclamer 
Pour les copies, les notaires réclament 6 deniers (ou pences de la livre anglaise, cours d'Halifax) par 100 mots. Dès qu'ils se déplacent, ils exigent $7 / 6$ par jour en frais, sans compter leur rémunération. ${ }^{205} \mathrm{Si}$ l'on exclut de nos calculs la présence indispensable d'un second notaire dans un nombre relativement important d'actes (actes par ailleurs coûteux, comme les inventaires après décès) ainsi que les copies supplémentaires de contrats précédents amassés dans les greffes, si on se restreint volontairement aux actes plus courts (ventes, homologations, quittances, etc., par opposition à des actes parfois très longs comme les inventaires après décès, les contrats de mariage, certaines donations, les partages ou les testaments), on peut conclure que le revenu minimum d'un notaire moyen ne saurait être inférieur à $£ 275 / 300 \mathrm{c}$. Il s'agit là d'une somme très supérieure à la plupart des emplois publics. ${ }^{206}$ Un notaire diligent ${ }^{207}$

\footnotetext{
davantage. (Cap. III, 1780, APC, M.G. 11, C.0. 44, 4 : 56-67.) D'autre part, les inventaires après décès, obligatoires pour la répartition des biens de la communauté dissoute, peuvent être très longs et occuper deux notaires durant plusieurs jours. Nous avons trouvé des inventaires de pius de vingt pages. Par contre, une quittance est généralement beaucoup plus courte, v.g. une page. Voici quelques exemples de comptes pour des actes plutôt courts (ils sont tirés des papiers Neilson et Taché, APC, M.G. 24, B. 1, vol. 176 et 183 , APQ, gr. coll., pap. Taché, b. 12) : acte de cession et d'abandon d'une terre à un seigneur et "échange" (1798) : 7/6; copie d'un contrat de vente d'une terre (1802) : 5/-; homologation d'un avis (1803) : recherche $2 / 6$, copie à $6 \mathrm{~d}$ les 100 mots $6 / 2$ et certificat $1 /-$, total $9 / 8$; contrat de vente (1808) : $£ 1.3 .4$; obligation (1808) : $7 / 6$; quittances (1808) : $3 / 6$ et 5/-; copie d'un contrat de vente de terre (1809): 12/6, plus un droit de $6 \mathrm{~d}$; bail à ferme $(1810)$ : recherche $2 / 6$, copie $3 / 9$, certificat $1 /-$, total $7 / 3$; contrat de mariage (1812) : £1.3.4.

205 "Notaries charge 25s. merely for making a protest: they are always accompanied by a brother notary, who receives $7 \mathrm{~s}$. $6 \mathrm{~d}$. for his walk, and for attesting the signature to the protest." (J. Lambert, Travels Through Lower Canada [...], 1: 204-205.). Quand ils agissent comme arbitres - rôle qui n'est pas un acte notarié, donc n'entre pas dans les registres d'actes proprement dits -, ils réclament la même somme, en plus de £1.1 par jour comme honoraires. Par exemple, un arbitrage en 1804 dure sept jours (APQ, gr. coll., pap. Taché, b.11 : s.p.).

206 Ce minimum a été calculé à partir du coût moyen de copies d'actes courts et moyens (5/- chaque copie, 4 parties en moyenne par contrat au moment de la signature) et d'une estimation du coût du contrat original au double du coût de la copie, ce que confirme d'ailleurs l'ordonnance de 1780 , soit 183 actes $\times 4$ copies $\times 5 /$ - plus 183 actes $\times 10 /$. Nous avons préféré ici un biais à la baisse à une estimation à partir de contrats plus longs et plus dispendieux ainsi que de tous les honoraires et frais afférents (déplacements, etc.).

$207 \mathrm{Il}$ est évident que certains notaires pratiquent peu, soit qu'ils sont incompétents, malchanceux ou malades, soit qu'ils diversifient leurs activités (notariat, commerce ou agriculture, politique, comme dans le cas de Papineau père). Leur moyenne parfois très basse (v.g. 15 ou 30 actes par année)
} 
peut gagner de $£ 400$ à $£ 1,000$. En demeurant conservateur, on peut donc affirmer qu'un notaire compétent et actif touche en moyenne de $£ 300$ à $£ 700$ c. par année dans le Bas-Canada, entre 1792 et $1812 .{ }^{208}$ Or seuls des juges, le grand voyer et le gouverneur reçoivent autant ou davantage. Et les notaires ne peuvent remplir ces fonctions, sauf celle de grand voyer.

La même situation semble prévaloir chez les avocats. Il est beaucoup plus difficile d'évaluer, même grossièrement, leurs revenus moyens. ${ }^{209}$ Cependant, diverses sources laissent soupçonner que loin de trop proliférer, ils sont débordés de travail. Eux aussi, on les inculpe de toucher des honoraires exorbitants et d'exploiter la complexité des lois canadiennes - des lois "féodales", au dire des Britanniques - pour escroquer la population. ${ }^{210}$ Chaque motion, chaque lettre, chaque papier quelconque, comporte son tarif, généralement de $2 /$ - à $10 /$ - pour les formalités routinières. Les avocats s'ingénient d'ailleurs à les multiplier. L'imprimeur John Neilson doit débourser $£ 2$ ou plus pour le recouvrement de petites dettes, la plupart du temps en bas de $£ 10$ ou $£ 5$. Dans des causes plus compliquées, l'avocat réclame $£ 5, £ 10$ ou même davantage. ${ }^{211}$ Les procès, au civil, nécessitent

déprime la moyenne générale. Par contre, d'autres notaires préparent de 250 à 625 actes par année (v.g. Louis Chaboillez à Montréal).

208 Cette sous-estimation brute devient peut-être plus réaliste, si l'on tient compte des dépenses des notaires (matériel de bureau, peut-être l'engagement d'un clerc) et des comptes perdus (bien que les notaires soient dans une position excellente pour exiger le paiement de leurs services). Mais toute profession, tout commerce nécessite un minimum de dépenses. En outre, la cléricature obligatoire des futurs notaires pourvoit les notaires établis d'une main-d'œuvre à bon marché qui permet de grossir les affaires.

209 Les avocats ne tiennent pas de répertoires de leurs causes. Nous n'avons pas retrouvé de livres de comptes suffisamment détaillés. Nous ignorons le nombre de causes au civil et au criminel par avocat, car trop de registres de cours ont disparu. Enfin, nous ne connaissons un large éventail d'honoraires dans les causes routinières que pour les années 1780 (ordonnance de 1780 fixant les honoraires des avocats et des notaires, APC, M.G. 11, C.O. 44, $4: 56-67$.$) . En 1810, les tarifs sont les suivants$ pour les cours inférieures, au civil : recouvrements de dettes entre 40/et $£ 5$ st. : $10 /-$; pour dettes de plus de $£ 5$ st. : $12 / 6$; pour poursuites en dommages personnels : 20/-; plaidoieries écrites : $5 /-$ en sus; dans des causes de plus de 40/- impliquant des obligations ou des notes promissoires : 5/- ("New Tariff of fees . .. 12th March 1810", Montréal, APC, M.G. 24, B. $1,16: 52-54$.$) .$

210 "Their fees are high ..." (J. Lambert, Travels Through Lower Canada [...], 1: 204; The Quebec Mercury, 23 mars 1807).

211 Voir APC, M.G. 24, B. 1, vol. 184 et 187; APQ, gr. coll., pap. Taché, b.11; APQ, seign. de Beau Rivage, livre de comptes d'un avocat (incomplet). Il faut payer des frais de cour relativement élevés (v.g. 
parfois la collaboration de plusieurs avocats pour une même partie. ${ }^{212}$ L'ignorance de la plupart des juges britanniques en matière de droit canadien ne peut que prolonger indûment les procès, ce qui décuple encore les frais légaux.213

L'expansion démographique, la restructuration et la diversification de l'économie, la multiplication des lois et la montée de la criminalité urbaine à la fin de la période, ${ }^{214}$ semblent avoir suffisamment élargi la base d'opération des professionnels pour éponger leur nombre croissant. ${ }^{215}$ Outre les avocats et les notaires,

$£ 15.8 .4$ par rapport à £11.13.4 d'honoraires dans une cause de 1812, APC, M.G. 24 , B. $1,16: 59$.), $£ 1.6 .8$ par jour pour la présence du juge, $£ 1.3 .2$ par jour pour celle du shérif, de 9/- à 13/- par jour ou davantage pour celle d'un témoin (ibid., $16: 53-54$ et passim). L'avocat touche généralement de $10 /-$ à $£ 2.10 .0$ pour le recouvrement de petites dettes (ibid., vol. 183 et 187) : "Advocate's fees for prosecuting claim till judgment" : £2.11.0 (1804), £2.10.0 (1810). Voici deux exemples de comptes d'avocat. Le premier est envoyé à John Neilson (10 juin 1799) : "I. John Neilson vs J. Antrobus : jugement interlocutoire : 0.3 .0 ; port de deux lettres : 0.1.2; honoraires : 0.10 .0 . II. Idem vs J. Antrobus : sommation : 0.3 .0 ; signification : 0.2 .0 ; entrée : 0.1 .0 ; règle contre le défenseur pour venir prêter serment : 0.1 .0 ; signification : 0.1 .0 ; honoraires : 0.10 .0 . III. Idem vs J. Antrobus : sommation et entrée : 0.5 .6 ; signification : 0.8 .0 ; honoraires : 0.10.0. IV. Idem vs L. Dumoulin : sommation et entrée : 0.4 .0 ; signification : 0.6 .0 ; honoraires : 0.10 .0 ; honoraires pour faire le recouvrement de $£ 2.10 .0$...: 0.5.0.” (ibid., 183 : s.p.). Compte de l'avocat J.-L. Borgia, un membre en vue du parti canadien, pour une cause qui dure du $1^{\text {er }}$ mai 1801 au 2 mai 1804 et qui rapporte à l'avocat £24.12.6 en honoraires divers, en plus d'entraîner des frais de $£ 12.1 .5$, soit un total de £36.13.11 en frais légaux (sans compter ceux de l'autre partie), tout cela pour un jugement de $£ 34.0 .31 / 2$ (!) en dommages : "1801. $1^{\text {er }}$ mai : instructions pour poursuivre : $0.6 .0 ; 26$ mai : projet de déclaration \& 2 copies : 0.10 .0 ; 30 mai : 4 transports : $0.4 .0 ; 3$ juin : attendances lors du retour : 0.3 .0 ; dresse comparution : 0.1 .0 ; motion pour appeler le défendeur : 0.3.0; motion pour filer 3 exhibits : $0.3 .0 \ldots 1804$. 12 avril : attendance 0.3 .0 et motion que la cause soit mise sur le rôle : $0.6 .0 ; 23$ avril : attendance remise : $0.3 .0 ; 14$ juin : attendance remise : $0.3 .0 ; 16$ juin : attendance remise : 0.3 .0 ..." Etc. (ibid., $17: 1-5$. .).

212 V.g. dans une dispute relative au testament de A. J.-Duchesnay (J.-P. Wallot, "Plaintes contre l'administration de la justice (1807)", $R H A F, 19$ (1965-1966) : 551-560; 20 (1966-1967) : 28-43, 281-290, 366-379.)' 213 Ibid.

214 Le gouverneur Craig attribue cette hausse de la criminalité à la prospérité (Craig à Liverpool, Québec, 17 mai 1810, APC, M.G. 11, Q. 112 : 173-176; aussi représentations des juges de paix de Québec et de Montréal, ibid., 176 sqq.).

215 A titre d'exemple, les Harts de Trois-Rivières, à la fin du XVIIIe et au début du XIX ${ }^{e}$ siècles, s'intéressent au commerce d'exportation et d'importation, vendent en gros et au détail sur le marché intérieur, exploitent des industries (brasserie, bois, moulins) et des seigneuries, spéculent sur des terres dans la zone seigneuriale et les cantons, etc. Durant 
les médecins et les arpenteurs aussi s'en tirent apparemment très bien. ${ }^{216}$ Des bureaucrates au haut de l'échelle n'espèrent-ils pas restaurer une véritable aristocratie canadienne en la fondant sur les professions? C'est pour cette raison qu'en 1795, le juge en chef applaudit au rejet par le Parlement provincial d'un projet de loi qui aurait admis trois marchands ruinés (dont J.-F. Perrault, que Dorchester nomme finalement greffier à la cour de Québec) au barreau, en les exemptant des cinq années réglementaires de cléricature:

For in a province where land was a small value, where there were but very few opulent persons, and the rigour of whose climate was such that no persons of wealth would come purposely to settle in it, how was the aristocracy to be supplied from among the natives better than by those who had been successful in a liberal profession, but what person of education or sentiment would engage in it unless the practice were restricted to those who had dedicated their money, time and labour to acquire the proper attainments. ${ }^{217}$

De fait, plusieurs seigneurs pratiquent une profession. Deux d'entre eux font même partie du groupe de leaders dans le parti canadien. ${ }^{218}$

Loin de reluquer les postes de la liste civile, les professionnels seraient plutôt tentés de les refuser parce qu'ils gagnent

cette période, ils ont recours à 97 avocats et 31 notaires différents dans les trois districts de la province, pour un total de plus de 1,000 pièces légales (voir Archives du Séminaire de Trois-Rivières, papiers Hart, L-b, L-c, L-d, L-e). Le seul de Tonnancour, un avocat, réclame $\$ 42.7 .19$ pour 48 actes légaux (surtout des recouvrements de petites dettes), entre mars et octobre 1810 (ibid., L-d-77). Parmi les avocats de Hart, on remarque Pierre Bédard, le chef du parti canadien, et $\mathbf{A}$. Berthelot, l'un de ses lieutenants. 216 Les comptes de médecins retrouvés dans divers papiers familiaux paraissent plutôt exorbitants. Par exemple, le docteur Longmore demande £27.13.4 (médicaments et visites) pour avoir soigné le frère aîné de John Neilson, mort en 1793 après une courte maladie (APC, M.G. 24, B. 1, 70 : 18 mars). Les papiers Hart contiennent aussi des comptes du même genre. En ce qui a trait aux arpenteurs, les concessions innombrables dans les cantons, entre 1796 et 1812 , constituent une mine d'or. En fait, le gouvernement prétexte de leur petit nombre pour expliquer la lenteur des concessions. Leurs honoraires sont fort respectables : 15/- par jour pour un arpenteur, $7 / 5$ pour un dessinateur. Voir APQ, arpentage, QBC-23, t. 3 (papiers Holland) et t. 4 (papiers Bouchette).

217 Le juge en chef Osgoode à King, Québec, 23 mai 1795, APC, M.G. 11, C.0. $42: 10$.

218 Jean-Antoine Panet et Jean-Thomas Taschereau, son gendre et le fils par surcroît du grand voyer du district de Québec et aussi surintendant des maisons de postes provinciales. Taschereau combattra pourtant le gouvernement et goûtera à la prison, en 1810, avec Bédard et Blanchet. 
bien leur vie. En 1800, le lieutenant-gouverneur Milnes recommande vivement une augmentation considérable (50 pour cent) de salaire pour les juges des cours du banc du roi : non seulement la hausse rapide du coût de la vie la rend-elle nécessaire, mais les avocats de quelque valeur dans la province font beaucoup plus que $£ 500$ st. par année; ils refuseront done de quitter leur pratique pour accéder au banc et le gouvernement devra combler les vacances par des hommes de moindre valeur. ${ }^{219}$ Effectivement, Jean-Antoine Panet, le président de l'Assemblée et, après Bédard, l'homme le plus important au sein du parti canadien, a dédaigné une place de juge, puis deux autres postes que lui offrait Dorchester en 1795 - au grand scandale des Britanniques.220

Cette conjoncture généralement favorable pour les professionnels comble également les chefs du parti canadien. Panet, co-seigneur il est vrai, vit dans l'aisance, entouré de cinq ou six domestiques (filles de chambre, cuisinières, garçons, précepteur pour chaque enfant et nourrice à chaque bébé).$^{221}$ Bédard mène unc vie professionnelle très active et son nom apparaît fréquemment dans les papiers judiciaires. ${ }^{222}$ Louis-Joseph Papineau commence à peine sa carrière professionnelle et politique qu'il se plaint d'être surmené et d'écrire ses lettres de nuit, "sur le temps du travail": "J'ai eu beaucoup plus d'ouvrage que je ne m'y attendais, je n'en suis pas fâché - il faut apprendre à travailler, à pourvoir à mes besoins . .." ${ }^{223}$ Le docteur Blanchet, l'un des prisonniers politiques de 1810, pourrait s'aiguiller sur la voie rapide des promotions: en 1802, il épouse en effet la fille du seigneur, conseiller et pensionné Antoine Juchereau-

219 Milnes à Portland, Québec, 8 mars 1800, APC, M.G. 11, Q. 84 : 172-173. Londres consentira à une hausse de $£ 500$ à $£ 750$ par année (Portland à Milnes, Whitehall, juillet 1800, ibid., 290).

220 "During all this time Mr. Panet's Commsn remained in the office waiting for his acceptance of it - an indignity to which I trust the King's Comsn's are not often exposed. After four months had expired it was withdrawn and given to another. About a week after ... [the] vacant employ of Solr. Gen. and Inspector of the King's Domains was offered by message from his Lordship [Dorchester] to this same Mr. Panet and refused in terms of such insolence that the secretary declined to return his answer." (Osgoode à King, Québec, 23 mai 1795 , secrète et très confidentielle, APC, M.G. 11, C.0. 42, $22: 8$.).

221 APQ, gr. coll., livre de comptes de J.-A. Panet (1800-1812). Panet a des clients britanniques ( 8 sur 30 en octobre 1806).

222 V.g. J.-P. Wallot, "Plaintes contre l'administration de la justice (1807)", 282 sqq.; APQ, QBC-28 (justice). Voir aussi note 197.

223 L.-J. Papineau à sa sœur Rosalie, 17 octobre 1811, APQ, gr. coll., papiers Papineau-Bourassa, b. 5 : lettre $n^{\circ} 138$; aussi idem à son père, Montréal, 26 octobre 1811, b. $4: \mathrm{n}^{\circ} 123$.). 
Duchesnay.224 Pourtant, il devient l'un des lieutenants de Bédard et un adversaire acharné de l'Exécutif. Maints professionnels canadiens-français réussissent aussi bien.225 En général, ils ne s'enrichissent pas, mais ils vivent bien de leur profession. On ne saurait en dire plus de ceux qui œuvrent dans les emplois publics: à preuve les lamentations de Ryland, qui cumule pourtant plusieurs bons postes. ${ }^{226}$

Dans leur ensemble, les professionnels n'empruntent donc pas la filière rapide. De plus, ils ne semblent pas avoir intérêt à convoiter les postes publics relativement mal rémunérés. Enfin, le parti canadien n'a pas cherché à multiplier les emplois et le patronage sous son contrôle grâce à des lois spéciales de l'Assemblée, comme il aurait pu le faire. Ce procédé laisserait intact le fief de l'Exécutif (la liste civile proprement dite). Les Assemblées de la Nouvelle-Ecosse et du Nouveau-Brunswick y ont recours à la même époque. H.T. Manning prétend que les chefs canadiens s'en abstiennent par ignorance. ${ }^{227}$ Mais alors, comment expliquer qu'ils suivent de très près ce qui se passe dans une colonie aussi éloignée que la Jamaïque, ${ }^{228}$ qu'ils reçoivent et lisent les Journaux des Assemblées des Maritimes, en plus d'ail-

224 Duchesnay annonce au comte des Méloizes le mariage de sa fille Henriette au "Docteur Blanchet qui n'a d'autre fortune que de grands talents et un très riche caractère et beaucoup de sagesse, qui, si je ne me trompe, la rendra la plus heureuse" (lettre de novembre 1802, APQ, gr. coll., lettres au comte des Méloizes). Blanchet, en 1802, vient d'arriver des Etats-Unis où il a complété ses études de médecine et publié un volume (Recherches sur la médecine).

225 C'est le cas de l'avocat Olivier Perrault, qui finira dans le camp des bureaucrates. En 1807, Perrault l'aîné se réjouit de sa grosse clientèle, mais regrette qu'Olivier ne divise pas son travail entre plusieurs clercs. "Tu dois te comparer à un manufacturier intelligent \& actif qui plus il a d'ouvriers à son service, plus il gagne, plus il fait d'affaires, plus il en fait avec promptitude et plus il augmente l'affluence des pratiques à son office - par la satisfaction qu'un chacun retire d'être servi avec célérité $\&$ exactitude. Les Anglais entendraient bien ce que je veux dire. Mais vous autres Canadiens ne le concevez pas, deux ou trois clercs vous embarrassent et vous cherchez midi à quatorze heures. Faites donc des avances pour cela et vous en retirerez un profit considérable." (Perrault l'aîné à Olivier Perrault, Rivière-Ouelle, 21 septembre 1807, APC, M.G. 22/6, 5-1 : 116-117.).

226 Voir APC, papiers Ryland, M.G. 24, B. 3 et R. Christie, History of [...] Lower Canada, $6: 297$ sqq.

227 H. T. Manning, "The Civil List of Lower Canada", 32 sqq.

228 "I can assure your Lordship that the example afforded them by the proceedings in the Island of Jamaica [où l'Assemblee s'est opposée au gouverneur avec succès], is not lost on them." (Craig à Liverpool, Québec, 18 mai 1810 , APC, M.G. 11, Q. 112 : 188.). 
leurs de consulter ceux des anciennes colonies américaines. Peuvent-ils vraiment ne pas connaître des exemples aussi proches qui leur permettraient d'assouvir leur supposé appétit de "beurre"? Cela ne paraît guère raisonnable.

Ces interprétations particulières des affrontements autour de la liste civile ne résistent pas, semble-t-il, à un examen un peu sérieux. Il faut explorer d'autres niveaux pour atteindre les motivations derrière ces tiraillements.

\section{Une hypothèse plus intéressante}

Lorsqu'ils dénoncent violemment l'accaparement des places par une petite clique, les chefs politiques canadiens voient plus grand. D'ailleurs Craig lui-même, en 1810, s'écarte de l'interprétation simpliste du Mercury ("l'odeur des cuisines") et suggère qu'ils ambitionnent, par le contrôle de la liste civile, "d'exercer ainsi ... un ascendant complet sur le pays". Le gouverneur se dit d'ailleurs corroboré dans ce jugement par les principaux Britanniques dans la colonie. ${ }^{229}$ Donc, si i'on a pu prétendre que l'Assemblée du Bas-Canada retarde sur celle des Maritimes en ce qu'elle n'exploite pas toutes les chances de créer des emplois spéciaux, ses revendications s'avèrent plus globales. Le parti canadien, au nom de la majorité canadienne, requiert en fait la responsabilité ministérielle et l'entière responsabilité fiscale, sous la seule restriction du statut colonial. La thèse de Craig paraît finalement plus logique et plus conforme aux faits: une sorte de lutte pour le pouvoir global se livre autour de la part aux places. ${ }^{230}$

Cette hypothèse nous semble plus riche et se conforme mieux aux aspects politiques, économiques, sociaux et ethniques de la liste civile que nous avons évoqués plus haut. Elle permet surtout

229 Voir section IV, surtout pp. 385-388. Entre 1808 et 1811, l'ensemble des dépêches de Craig décrivent les ambitions (réelles ou imaginaires) individuelles et sociales, démocratiques, constitutionnelles et nationales des chefs canadiens. Voir J.-P. Wallot, Le Bas-Canada sous l'administration de Craig (1807-1811), thèse de doctorat manuscrite, Université de Montréal, 1965.

230 Une remarque de Bédard, en 1814, vient confirmer le jugement de l'ancien gouverneur : "Une remarque qui montrerait bien que le parti qui est en possession de l'administration a pour principe de ne recommander aucun Canadien à moins qu'il ne leur soit dévoué, c'est que Mr Panet l'orateur de la chambre depuis tant de temps n'a pas été fait conseiller exécutif, sans qu'il en parait d'autre raison sinon qu'il est attaché aux Canadiens." (Pierre Bédard à John Neilson, Trois-Rivières, 17 février 1814, APQ, gr. coll., papiers Neilson, b. 1: s.p.). Titulaire lui-même d'un emploi, Bédard songe à l'essentiel, à la source du pouvoir : à l'Exécutif. 
de prendre une vue des conflits autour de la part aux places qui intègre la multiplicité des niveaux et des groupes sans en retrancher aucun à priori. Que dire, par exemple, des classes non professionnelles qui forment l'immense majorité de la population et le gros des députés canadiens-français: ce sont eux qui accordent un appui indéfectible aux leaders canadiens et qu'intéresse au premier chef la possibilité d'avoir accès aux postes de la liste civile. Ceux-là - "habitants indépendants", individus à revenus modestes et armés d'un minimum d'instruction, organisateurs électoraux qui parfois font élire les professionnels in absentia, artisans, maîtres d'écoles, bref la petite bourgeoisie qu'ignorent l'historiographie et la masse - lorgnent les emplois subalternes. Leurs motivations ne participeraient que de loin à une volonté abstraite de pouvoir. Ils se satisferaient tout aussi bien de ces lois spéciales que l'Assemblée pourtant ne vote pas.

Imaginer que les professionnels canadiens luttent contre l'ancienne noblesse et la bureaucratie britannique afin de leur arracher des places et de s'y caser eux-mêmes, c'est simplifier à outrance. Cette ambition mesquine ne suffit pas à expliquer le militantisme persistant des chefs canadiens et, derrière lui, celui de la petite bourgeoisie et du peuple. Nous voudrons lire dans les motivations des classes moyennes une mixture de ce patronage paternaliste et de patronage plus proprement politique axé sur l'accès au pouvoir..231

L'aristocratie canadienne, pour sa part, se débat avec d'autant plus d'acharnement qu'elle est en pleine décadence. C'est la classe que frappent le plus durement les renversements dans les politiques de pensions et d'emplois (voir section V): les professionnels n'en ont jamais détenu qu'un nombre infime. Privée de l'assise économique qui la maintenait en place sous le régime français, dépendante du gouvernement pour les quelques faveurs et places qui lui sont d'ailleurs comptées, face par conséquent à un éventail plus restreint de débouchés et à une concurrence accrue d'autres groupes lors même que ses effectifs se gonflent rapidement, méprisée enfin du peuple à cause de son servilisme et de ses exigences seigneuriales accrues,

231 Dans ce contexte, "even seemingly insignificant office proved attractive to those who were not so much interested in the office itself as in the further patronage power it might control, or as a means of satisfying the wants of some lesser "connection'" (R. Shipkey, "Problems of Irish Patronage during the Chief Secretaryship of Robert Peel, 18121818", The Historical Journal, 10 (1967) : 42.). Il s'agit d'un patronage véritablement politique. Voir note 56 et Alex Weingrod, "Patrons, Patronage, and Political Parties", 377 sqq. 
elle déchoit sur les plans économique et social. ${ }^{232}$ Sans influence politique au niveau des élections et de l'Assemblée, c'est comme minorité qu'elle s'allie aux Britanniques dans les Conseils. Il ne faut donc pas se surprendre de ce que les jeunes aristocrates déploient autant sinon davantage d'énergie à contester les ambitions de la nouvelle élite professionnelle qu'à enrayer les projets assimilateurs des Britanniques militants. ${ }^{233}$ Face à un statut et à un pouvoir qui s'effritent, l'aristocratie régresse vers des formes de patronage plus paternalistes. Ses réflexes sociaux et politiques démasquent un groupe social clos sur lui-même, ${ }^{234}$ qui exhalte des

232 "Dévalorisation économique, militaire et sociale, tel sera de plus en plus le lot des seigneurs." (F. Ouellet, op. cit., 567). Voir les témoignages de Nepean, en 1789 ( DC I, 942 sqq.), de Dorchester, en 1794 (APC, M.G. 11, Q. 69-1 : 1 sqq.), d'Osgoode, en 1795 et 1796 (ibid., C.O. 42, 22 : 8 sqq., 53-54), de Weld, en 1799 (Travels Through the States of North America [...] (Londres, 1799), 230), de Milnes, en 1800 (DC II, 228-245), de Lambert, en 1808 (op. cit., 1: 198-199), et de Craig, en 1808 et 1810 (APC, M.G. 11, Q. 107 : 195 sqq.; DC II, 392-405).

233 Par exemple, dans Le Courier de Qúévec. Vơir J. Hãre et J.-P. Wallot, Les Imprimés dans le Bas-Canada, 1801-1810, 332-344.

234 L'un des signes de cette sclérose sociale, c'est la chasse obsessionnelle aux fortunes grâce à des mariages entre nobles ou avec de riches roturiers (rières) - notamment entre filles de seigneurs et marchands britanniques. Cette recherche prioritaire de bons partis désavantage même les cadets des familles seigneuriales. Vers 1790 , F. Cugnet écrit à de Lavaltrie: "N'avez-vous pas déjà vu les provisions américaines permises pour l'importation - Ils [les Américains] vont sans doute nous amener jusqu'à leurs filles, comme provision fraîche, et je vous assure que leur qualité reconnue d'aimer les étrangers nous réjouit ici tous Cavaliers que nous sommes. Car du moins aurons-nous espérance de ne point vieillir si longtemps dans le triste état de célibataire, dans lequel plusieurs languissent depuis que les parents canadiens ne veulent marier leurs filles non suivant leurs inclinaisons, mais suivant une rigoureuse convenance et une rigide égalité de fortunes..." (APC, M.G. 22/6, 2: 169.). Duchesnay note "la disette de partis bien nés" et consent pour cette raison, au mariage d'une de ses filles avec le seigneur Taschereau, de beaucoup plus âgé qu'elle. (A. de Juchereau-Duchesnay au comte des Méloizes, Beauport, 23 août 1789, APQ, gr. coll., lettres au comte des Méloizes). Les seigneurs ne dédaignent pas les bons partis roturiers pour eux-mêmes (v.g. Chaussegros de Léry et Chartier de Lotbinière épousent des filles de juges britanniques. Voir ibid.). Par contre, une jeune fille bien née, mais sans fortune, écope d'un lourd handicap (Ch. de Lotbinière à son oncle, Québec, 9 juin 1796, APQ, gr. coll., papiers Chaussegros de Léry, b. 8: s.p.). Les Salaberry, en Angleterre, songent à marier de bons partis. Et François-Louis se réjouit du mariage de sa sœur à Juchereau-Duchesnay car "il aura la meilleure partie de la fortune de sa mère... J'espère que les deux autres demoiselles suivront ce bon exemple." (Lettre à son père, Madras, 25 juillet 1808, APQ, gr. coll., papiers Salaberry (copies), b. 1: s.p.; aussi b. 2: passim.). Pour Xavier de Lotbinière, le mariage de sa nièce à un simple habitant "déshonorerait" autant la nièce que toute la famille: “... s'il fallait qu'un braceux comme cela entre dans sa famille... elle pleurerait nuit et jour, 
valeurs d'un autre temps..$^{235}$

Les Britanniques ou les premiers bénéficiaires du système, de leur côté, bloquent les revendications canadiennes pour un grand nombre de raisons. Ils redoutent l'Assemblée, trop démocratique à leurs yeux. ${ }^{236}$ Ils rembarrent le projet de la "nation canadienne" et s'en tiennent à une stricte autorité de l'Exécutif sur les finances publiques, Exécutif où ils conservent la majorité. A leur avis, les Canadiens représentent à tort qu'ils essuient des injustices dans la distribution des "faveurs" gouvernementales. Tant qu'ils s'inspirent d'une langue, de lois et de principes français, peuvent-ils s'attendre à ce que les Britanniques leur décernent un traitement de faveur, leur confient des postes de confiance dans le gouvernement et les préfèrent à leurs propres nationaux et amis? ${ }^{237}$ Les Canadiens revendiquent une part plus grande des places et se plaignent des distinctions entre eux et les Britanniques en ce domaine. C'est leur faute: "They keep alive the distinction by their tenacity... it is requisite they should identify themselves with the people [les Britanniques] of whom they are now a permanently established part. Without this they must be ever more in a subordinate sphere." ${ }^{238}$ Tant

l'expérience ayant de tout temps montré que ces gens-là ne veulent que la perte des gens bien nés où ils entrent..." (Lettre à Gaspard de Lanaudière, Québec, 25 avril 1812, APQ, gr. coll., papiers Tarieu de Lanaudière (copies), b. 2: s.p.; même à la veuve de Gaspard, 25 juin 1812 , ibid.). Une jeune fille instruite se lamente à deux reprises dans Le Courier de Québec que les parents canadiens forcent désormais leurs enfants à se marier pour des "raisons de convenance", qu'ils assortissent "les fortunes \& non les cours" et trafiquent leurs filles (numéros du 27 février et du $1^{\text {er }}$ octobre 1808). 235 Voir note 234. Voici deux autres illustrations de cette attitude. Agathe de Lanaudière invite Gaspard de Lanaudière à se présenter aux élections, même s'il risque la défaite: “....c'est absolument nécessaire que les personnes comme il faut se montrent... c'est le devoir de tous les hommes qui sont attachés à leur roi de faire cet effort... si tu ne réussis pas, tu auras fait ton devoir pour ton pays, à Dieu..." (lettre du 2 avril 1810, APQ, gr. coll., papiers Tarieu de Lanaudière, b. 2: s.p.). Au début de la guerre de 1812 , $X$. de Lanaudière raconte qu'il est occupé du matin jusqu'au soir sans répit, "mais je suis dans une office de gouvernement de conséquence [en fait, il est traducteur et secrétaire français du Conseil exécutif] et je veux y faire mon devoir en homme bien né et en bon sujet et voilà chère amie comment il faut agir et surtout en temps de guerre" (lettre à sa belle-sœur, Québec, 4 juillet 1812, ibid.). 236 V.g. [John Henry], An Enquiry into the Evils of General Suffrage [...] (Québec, 1810); The Quebec Mercury, 23 avril 1810. $237 \mathrm{H}$. Gray, Letters from Canada [...], 329, 103; The Quebec Mercury, 9 mars 1807.

238 The Quebec Mercury, 3 avril 1809. Voir aussi ibid., 9 mars 1805, 14 avril 1806, octobre, novembre et décembre 1806, janvier 1807, 27 avril 
qu'ils ne s'assimileront pas, les Canadiens se sentiront étrangers dans leur propre pays. ${ }^{239}$ Pourquoi sont-ils absents du commerce et des hautes fonctions? "The answer may be found in the policy of those who endeavour to preserve the Canadian character." ${ }^{240}$ Des Canadiens accusent d'ailleurs volontiers les promus de s'être anglicisés et protestantisés pour arriver. ${ }^{241}$

Le débat autour de la part aux places s'inscrit donc dans la lutte pour le pouvoir; et la ronde des groupes qui s'y croisent, chacun avec ses méthodes, sa vision du monde et ses objectifs, érige une sorte de hiérarchie qui n'est pas sans rejoindre certains malaises du système global dans lequel il s'insère.

1807, 31 octobre et 26 décembre 1808, 3 avril, 29 mai et 10 juillet 1809 , 12 et 19 mars, 16 et 23 avril 1810; APC, M.G. 11, Q. 113: 214-216; DC II, 392-417; etc.

239 R. Cuthbert, An A pology for Great Britain [...] (Québec, 1809), 29. 240 [John Henry], An Enquiry [...], 29 sqq.; J. Fleming, Some Considerations on this Question [...] (Montréal, 1810), 17 sqq.

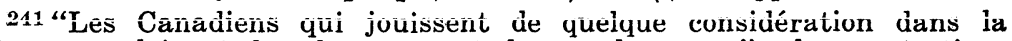
province, ne doivent le plus souvent leurs places qu'à des protections achetées bien cher et souvent au prix de la flatterie ou d'une dissimulation honteuse de leurs principes, de peur de heurter ceux qui dominent au-dessus d'eux... [et les tiennent] dans une servitude humiliante." (Mgr Plessis à l'abbé Bourret, Québec, 10 et 15 mai 1807, AAQ, registre de lettres, 6: 20 sqq.). Nous avons déjà vu ce que Le Canadien pense des "gens à places". Voir aussi M. Brunet, La Présence anglaise et les Canadiens [...], 99-100; B. Sulte, Histoire des Canadiens français (8 vol., Montréal, 1882-1884), $8: 31-32$. Les Britanniques eux-mêmes dédaignent certains des promus qui se comportent en parvenus ridicules. Après un voyage à Montréal en compagnie de Joseph Bouchette, nouvellement promu arpenteur général, Sewell livre ses impressions: “...my companion ... would talk of nothing else but her [madame Bouchette] - her relations - their fortunes, splendor and magnificences, his fears of her assuming new ideas, incompatible with his income, and his conviction of her being infallible - In short, he was continually loud in his fears $\&$ her praise, never breaking the thread of his discourse except to interrogate me on some important point as whether he should when at Montreal write on his card "Mr Bouchette" or "The Surveyor General", whether "He should wait on McTavish first or stay till he called on him" \&c \&c..." (Sewell à sa femme, 1er mars 1804 (copie), APQ, gr. coll., papiers Sewell). Le marquis Michel Chartier de Lotbinière se morfond aux Etats-Unis, de 1789 à 1798, dans le vain espoir d'obtenir un pardon du gouverneur - il a opté autrefois pour les révolutionnaires américains - et de venir mourir au Canada parmi ses enfants et petitsenfants. Son propre fils, un homme à place, n'ose en souffler mot au gouverneur qu'il rencontre souvent, de crainte de l'indisposer. Et le marquis meurt à New-York en 1798 de "fièvre jaune", selon son fils, et "à moitié fou" car il a vendu la seigneurie de Beauharnois "pour un morceau de pain"! (Michel Chartier, marquis de Lotbinière, au comte des Méloizes, New-York, 12 octobre 1789 et Gaspard-M.-A. Chartier de Lotbinière au même, Vaudreuil, 8 septembre 1802, APQ, gr. coll., lettres au comte des Méloizes). 


\section{La liste civile et le malaise au niveau global}

On peut découper tout ensemble, si restreint soit-il, en deux segments: les parties qui le composent et les relations entre ces parties. Jusqu'ici, nous avons décanté certaines observations à partir d'une analyse de la liste civile comme tout, et ausculté les motivations des divers groupes qui constituent en quelque sorte les parties de cet univers économico-politique. Or, il faut éluder le danger que nous avons noté plus haut: celui de prêter certains traits du système à l'action d'une de ses parties. L'idée d'une influence asymétrique de l'une des parties sur toutes les autres n'est pas à exclure à priori; mais certains travers du système peuvent être aussi bien imputables au treillis de relations qui soudent ces acteurs en une situation concrète. En effet, il faut se rappeler que "... le tout est différent de la somme des parties : il constitue un système général dont la dynamique propre et l'excellence dépendent autant des relations entre les parties que des parties elles-mêmes". ${ }^{242}$

Il faut donc distinguer clairement les motivations et les actions des équipes particulières autour de la liste civile, de la ronde dans laquelle elles s'engrènent. Une série de groupes rationnels poursuivant leurs intérêts peuvent fort bien ne pas s'agréger en un tout harmonieux; et certain malaise ressenti au niveau du tout, peut esquiver les tentatives d'explication si l'on ne se situe d'emblée au niveau de l'examen des relations qui sous-tendent cet univers global.

Or, l'étude de la liste civile débusque un certain nombre de ces facteurs relationnels qui se font jour à tous les tournants de l'analyse. Par-delà les aspects institutionnels et les intérêts mesquins d'une ou de classes sociales, bien incapables à eux seuls d'embrasser toute la réalité, les relations ethniques ellesmêmes n'épuisent pas le problème du patronage, il s'en faut, bien que les éléments constitutionnels, sociaux et ethniques, et leur interaction, conditionnent dans une large mesure la portion spécifique de parties et de relations que concrétise la société bas-canadienne. Mais, aux fins de notre enquête, nous nous attacherons à deux facteurs relationnels plus généraux: la relation impériale et le réseau des communications entre groupes. Ces relations représentent respectivement les dimensions externe et interne de la liste civile comme institution. Selon nous, une

242 G. Paquet, "Vers une médecine rationnelle", Le Médecin du Québec, 5 (mars 1970): 23; aussi G. J. Klir, An Approach to General Systems Theory (New-York, 1969). 
bonne part du malaise signale surtout dans la section IV mais qui transparaît aussi dans nos analyses des sections V et VI, émane de ces phénomènes relationnels. Plus encore, ces derniers nous semblent revêtir le même poids relatif dans la société bascanadienne que dans l'univers économico-politique de la liste civile. Nous verrons brièvement si l'on ne pourrait pas attribuer à ces composantes une portion congrue du malaise global qu'a étudié l'historiographie conventionnelle.

a) Le clivage ethnique dans le combat autour de la part aux places n'a rien de surprenant. Moins encore quand on a compris qu'il s'agit d'une lutte pour le pouvoir et si l'on se souvient que les métropolitains sont britanniques et la masse des coloniaux, canadiens-français. En effet, la métropole veut exploiter les ressources de sa colonie. Elle tend donc à installer une administration qui lui assure le contrôle effectif de la vie économique et de la société coloniale. La dynamique de l'ajustement général entre métropolitains et coloniaux est justiciable d'une analyse sociologique que ccrtains ont déjà amorcée. Ainsi, Trevor Denton soutient que les relations conjonctives entre métropolitains et coloniaux d'abord, puis entre coloniaux anglais et francais, sont telles qu'elles tendent "to discourage any assimilation during the period from the Conquest to roughly $1800 " .{ }^{243}$

La relation métropole-colonie infléchit tous les rapports sociaux et politiques entre groupes autour de la liste civile. Les affrontements anglais-français, le biais dans la sélection et la promotion pour les postes clé, rien de plus normal dans le contexte qui nous occupe. L'état d'infériorité de la masse des coloniaux ne se manifeste pas seulement dans la liste civile, mais transparaît encore dans les autres catégories d'emplois semi-publics et sur le plan militaire. ${ }^{244}$ La suppression du Corps des volontaires royaux canadiens, en 1802 , ne peut que resserrer davantage les rares canaux de promotion. Plus grave cependant est leur disparition du commerce. Sans doute peut-on interpréter diffé-

243 "While there were some early British agricultural interests, many of the British who first came to Quebec were interested in running the flourishing fur trade. French Canadian merchants were kept out of the fur trade and indeed from most specialized economic activity which was not of an agricultural nature. This fact resulted in the rather surprising unintended consequence of a boundary-maintaining mechanism imposed by the British on the very people whom they wanted to assimilate." (T. Denton, "The Structure of French Canadian Acculturation", Anthropologica, 8 (1966) : 34-35.).

244 Voir notes 57 et 66. 
remment les causes de cette déchéance économique. ${ }^{245}$ On ne peut nier le phénomène. Les rares Canadiens français qui participaient encore au grand commerce, vers 1785-1795, en sont disparus ou sont morts au début du XIX ${ }^{\mathrm{e}}$ siècle. Et l'ensemble des Canadiens français, y compris leur élite, doivent se faire ou demeurer colons, gratte-papiers, ou au mieux, professionnels et clercs. L'agriculture, les professions libérales et l'état ecclésiastique constituent de plus en plus leur horizon économique, social et mental, hormis pour l'action politique à l'Assemblée, Chambre d'ailleurs contenue de tous côtés par des freins efficaces. Il reste bien une autre solution: l'exil. Un grand nombre de Canadiens, probablement de toutes les classes, ${ }^{246}$ s'expatrient aux Etats-Unis ou en Europe soit pour étudier, soit pour tenter fortune. Dans ce dernier cas, beaucoup semblent végéter ou échouer lamentablement. ${ }^{247}$ Mais il n'y a pas davantage d'avenir au Canada pour les jeunes Canadiens bien nés et ambitieux. Le lieutenant-gouverneur Milnes se préoccupe d'ailleurs, comme ses prédécesseurs et successeurs, de la pauvreté des seigneurs et de l'absence d'une

245 Pour les uns, l'élimination économique graduelle des Canadiens s'avère une conséquence inéluctable de la Conquête et de la substitution d'une métropole comme d'une élite britanniques à une métropole et une élite françaises (voir M. Séguin, La Nation "canadienne" et l'agriculture (1760-1850) (Trois-Rivières, 1970), conclusion; G. Frégault, La Guerre de la Conquête (Montréal et Paris, 1955), conclusion; M. Brunet, La Présence anglaise et les Canadiens (Montréal, 1958), 49-166). D'autres expliquent cet affaissement par l'inadaptation de la bourgeoisie canadienne-française aux réalités nouvelles de l'économie et par ses valeurs d' "Ancien Régime" (voir D. G. Creighton, The Empire of the St. Lawrence, 21, 33-34, 126-127, 160 ; A. R. M. Lower, Colony to Nation (Toronto, 1947), 66-69, 106-110, 124-126; F. Ouellet, op. cit., 539-596). Notre propos ici est moins de choisir entre ces interprétations que de poser clairement la dimension coloniale du Canada comme fragment d'un univers impérial très vaste, avec les contraintes qui en découlent sur sa structure et son fonctionnement. Dans ce contexte général, la dimension ethnique est accidentelle.

246 Les fonds privés des APC et des APQ contiennent la correspondance des grandes familles canadiennes (v.g. les de Salaberry, de Léry, de Lotbinière, de Lavaltrie, de Lanaudière), disséminées aux quatre coins du globe. Nul ne saurait comprendre la mentalité de ces familles sans tenir compte de cette dispersion plus ou moins forcée. D'autre part, on connaît plusieurs cas d'émigrés non nobles, mais instruits. Mais on connaît très mal le cas des simples Canadiens qui ont quitté le Bas-Canada pour le Haut-Canada, l'Ouest, les Etats-Unis, la France ou ailleurs (v.g. des marins), car ils ont laissé peu de lettres. Toutefois, par divers témoignages qu'il serait trop long de grouper ici, on sait qu'il s'en trouve un certain nombre.

247 Voir l'opinion de F. Blanchet sur les Canadiens vivant à New-York (Blanchet à Neilson, New-York, 11 août 1800, APC, M.G. 24, B. 1, 1 : 222-224.). Nous verrons plus loin le cas de Georges de Léry. Pour celui du marquis de Lotbinière, voir APQ, gr. coll., lettres au comte des Méloizes. 
aristocratie locale bien dotée. ${ }^{248}$ L'un des membres de cette aristocratie a bien exposé cette situation depuis son exil:
J'avoue qu'il est un peu embarrassant de prendre un parti dans la position où nous sommes. Il n'est pas gracieux d'avoir à choisir entre le Canada et une campagne dans l'armée de Condé .... si je te demandais quelle ressource on pouvait espérer du Canada il me semble que là dessus tu n'as pas de grandes espérances. L'état de notre fortune ne nous permet cependant pas de rester oisifs. Ce n'est que par le travail et la patience que nous pouvons nous mettre au-dessus de nos affaires. Je ne crois pas que les héritages puissent y contribuer beaucoup encore moins les mariages. Il faut donc user d'in- dustrie. Que peut-on espérer de notre cher pays. Charles est placé mais je préférerais être enterré tout vif au fond d'une campagne, que de faire le métier qu'il fait. ${ }^{249}$ On a assez de ses propres sottises à débiter sans se rendre l'interprète de celles des autres. Quel emploi convenable voudrait-on te donner, ou ne te resterait-il qu'à te mettre sur une terre pour la faire valoir ... Il me semble que dans un pays encore neuf comme le Canada, on pourrait, avec de l'industrie et de la conduite, se former à la campagne un petit bien qui suffirait à mener une vie aisée et indépendante . . . Le Com- merce offrirait encore quelques ressources, mais sans fonds, il faudrait être courtaud de boutique ou aulner modestement un bout de ruban au premier monsieur en fourrole rouge qui se mettrait en dépense pour sa belle. ${ }^{250}$

248 Milnes à Portland, $1^{\text {er }}$ novembre 1800, DC II, 252-258.

249 Charles de Léry est assistant-greffier et traducteur du Conseil législatif (environ $£ 90$ st. par année).

250 Georges de Léry à son frère Louis-René, 30 novembre 1794, APQ, gr. coll., papiers de Léry, b. 10: s.p. (voir aussi notes 60 et 67). Georges ne réussira pas à faire fortune en Europe: il a choisi le mauvais parti au moment de la Révolution, celui des royalistes. Il se promène un peu partout, en Espagne, en Russie, en Lithuanie d'où il écrit en 1805 qu'il est toujours célibataire et pauvre (idem à idem, Grodno, Lithuanie, 10 et 22 mars 1805, ibid.). Il deviendra finalement précepteur des enfants du Tzar! Le cas de cette famille est particulièrement intéressant, car un autre frère, JosephFrançois-Charles, qui a opté lui pour la Révolution, se hisse au rang de général dans le Corps de génie. Par ailleurs, dans le Bas-Canada, leur père a tout fait pour trouver une place à Georges (J.-G. Chaussegros de Léry au duc de Kent, Québec, 24 mai 1796, ibid., b. 8: s.p.) ainsi qu'à ses autres fils, Charles et Louis-René (voir notes 60 et 67). Après sa mort, Louis-René invoque la coutume locale voulant que les fils succèdent à leurs pères pour solliciter une place honorifique (dans le Conseil législatif), "un pas pour avoir par la suite un emploi plus lucratif". Ce serait une façon de "soutenir par les bienfaits de S.M. une famille des plus attachées à son gouvernement et une des meilleures du pays qu'il est de l'intérêt et de la générosité du roi de ne pas laisser tomber en Canada" (Ls-R.-C. de Léry à King, 26 décembre 1797 , ibid., b. 10 : s.p.). 
La correspondance des Canadiens, en général, analyse lugubrement leurs embarras financiers. ${ }^{251}$ Certains, empêtrés dans les mailles trop resserrées de leurs dettes, fuient la province pour ne pas y expier leur pauvreté. ${ }^{252}$ Faut-il s'étonner alors du mécontentement parmi les Canadiens de toutes les classes? La gêne des seigneurs, par exemple, ne peut qu'affecter les censitaires qui voient leurs rentes s'accroître. Même des Britanniques sentent ce mécontentement:

Certain it is . . . that the bulk of the Canadian Inhabitants do not feel quite satisfied - and that not from any neglect on the part of the Government towards them, but from a spirit of jealousy of the preeminence which the British have acquired ... 253

Il transparaît non seulement dans les désordres populaires (v.g. en 1794, 1796), les campagnes électorales et un journal de combat comme Le Canadien, mais aussi dans l'organe des jeunes "gens à places", soit Le Courier de Québec, témoignage d'autant plus significatif puisqu'il vient des privilégiés de l'Etat. Or Le Courier,

251 V.g. l'immense collection Baby, aux APC, est truffée de telles plaintes. On pourrait en dire autant des papiers de Léry, aux APQ, etc. 252 Par exemple, Joseph Guy, obéré, doit s'enfuir aux Etats-Unis en 1807. Au moment même où les bourgeois britanniques s'enrichissent, les Canadiens à Détroit végètent. "Le commerce des blancs n'est pas trop brillant pour le présent", écrit Joseph Guy à son père, en 1800 (lettre datée de Détroit, 5 août 1800, APC, M.G. 22/6, 45-2: 663; voir aussi ses lettres du 25 janvier et du 17 février 1800, ibid., 647-648, 651). Hubert Lacroix reprend la mềme chanson en 1802 : le commerce est "de plus en plus mauvais" (H. Lacroix à J. Guy fils, Rivière-aux-Raisins, 20 janvier 1802, ibid., 21: 148.). Et encore, en 1805: “...tous les marchands ont perdu de l'argent cette année, et il y a encore apparence d'une mauvaise année... La misère va se faire ressentir dans toutes les parties du monde." (J. Guy à son père, Makinac, 3 juin 1805, ibid., 48: 29.). Son père lui répond que les affaires ne vont pas mieux à Montréal. Un marchand nommé Gilbert s'est même tranché la gorge, bien qu'on ignore si c'est à cause de mauvaises affaires ou d'une maîtresse (Pierre Guy à son fils, Montréal, 20 juillet 1805 , ibid., 34). L'année suivante, situation encore pire: "Tu me dis que l'argent est rare au Détroit, ce n'est pas le seul endroit: de ma mémoire, je ne l'ai pas vu si rare à Montréal avant la conquête que depuis: les marchandises aux encans se donnent, on ne peut pas dire qu'elles se vendent. Tous les jeunes marchands de ta connaissance ont été obligés d'abandonner le commerce." (Idem à idem, Montréal, 29 avril 1806, ibid., 25: 18.). Même si l'on fait la part des fluctuations de la conjoncture, ces témoignages illustratifs révèlent une crise endémique parmi les quelques marchands canadiens-français, à une époque où se construit le gigantesque empire de la Compagnie du Nord-Ouest et où se multiplient les maisons britanniques d'importation et d'exportation.

253 Major R. Lethbridge, 60e reg., à Townshend, 10 mars 1799, cité dans Sir L. Harmworth, The Northcliffe Collection (Ottawa, 1926), 429. 
tout en célébrant les vertus du commerce, condamne les commerçants britanniques qui veulent changer les mœurs des Canadiens et les gouverner à leur place: "Il faudrait leur laisser le soin de les conduire, et nous, rester spectateurs tranquilles et indifférents de leurs utiles et glorieux travaux." ${ }^{254}$ Si les Canadiens réussissent peu dans le commerce, ce n'est pas parce qu'ils parlent français, "mais bien plutôt parce que ceux qui en possèdent déjà tous les avantages, prennent ordinairement les mesures qu'ils savent devoir leur en assurer l'entière et exclusive possession." 255

Ainsi, le biais ethnique que nous avons cerné dans nos analyses nous apparaît en partie une conséquence du conflit normal métropolitains-coloniaux. Il s'agit là d'un travers dont le poids se fait sentir sur toute la structure économique, politique et sociale de la colonie - de toute colonie, même homogène. Le débat à propos de l'infériorité économique et sociale des Canadiens a souvent ignoré cette contrainte externe qui devait enrayer leur développement économique et social même lorsque les métropolitains étaient français plutôt que britanniques. ${ }^{256}$ Le fait que les métropolitains soient anglophones après 1760 complique le problème, mais a souvent permis de mieux percevoir ce qui existait pourtant auparavant.

b) Cette contrainte externe (la relation métropole-colonie) se double d'une autre qui la complète du dedans. Celle-ci, de caractère plus strictement économique, est fondée sur le problème des coûts de l'information - problème qui surgirait et qui a surgi à l'intérieur de colonies homogènes, ${ }^{257}$ mais qui accentue encore la torsion première si une masse considérable de

254 Le Courier de Québec, $1^{\mathrm{er}}$ avril 1807.

255 Ibid., 11 avril 1807.

256 Tant que le Canada n'aurait pas secoué le joug colonial (essentiel, bien sûr, à certains égards, surtout dans les débuts de la colonie), il aurait écopé du biais dont nous parlons. Sans doute, une petite minorité de coloniaux pouvaient accéder à des postes dans la colonie ou dans l'Empire, ce qui disparaît en bonne partie par la suite pour le segment canadien des coloniaux (voir b). Mais à côté des réussites, on oublie le grand nombre des Canadiens qui ne pouvaient bénéficier de filières largement tributaires de la métropole française.

257 Les hommes qui détiennent le pouvoir dans la métropole, nomment des amis, parents et alliés aux postes les plus importants dans une colonie. Ceux-ci exercent à leur tour un patronage pour les postes subalternes en privilégiant parents, amis et alliés (métropolitains et coloniaux). Il y a donc un réseau métropolitain qui projette un second réseau subalterne. Tous les coloniaux n'ont pas accès aux informations requises ni au même degré non plus qu'ils sont branchés sur le réseau. 
coloniaux (en l'occurrence, les Canadiens) se trouve coupée du circuit au départ. En effet, même après avoir fait la part du patronage des ministres et des hauts fonctionnaires londoniens, une autre portion du biais ethnique que nous avons noté à la section VI, est imputable à l'existence d'un réseau de communication anglophone à l'intérieur du Bas-Canada. Ce circuit est tel que le coût d'acquisition de l'information s'avère plus faible pour les postulants anglophones que pour les francophones. Ce phénomène se concrétise en une demande somme toute assez faible pour les membres des sous-groupes qui se trouvent hors du réseau de communication dans l'administration publique bascanadienne. ${ }^{258}$

Pour autant que les coûts d'acquisition de l'information ne sont pas négligeables et que l'administration doit les assumer, on peut s'attendre à ce qu'elle utilise la "filière" des moindres coûts: attitude tout à fait rationnelle. Or, même si ce facteur n'a pu jouer aussi fortement que dans la jungle des bureaucraties modernes où ces coûts de l'information ont acquis une pondération énorme, on peut croire que l'existence de ce double réseau de communications dans le Bas-Canada a contribué à biaiser la sélection en faveur des anglophones et contre les francophones.

On peut même avancer que ce travers, visible au niveau de la liste civile, est aussi l'une des caractéristiques de la société bas-canadienne tout entière. La structure dédoublée des classes vivant dans des réseaux de communications d'abord étanches, puis de plus en plus en coexistence hostile, a certainement contribué elle aussi à infléchir toute la série des rapports entre groupes. ${ }^{259}$ Mais il s'agit là d'un phénomène encore trop peu étudié pour que nous puissions en faire le tour maintenant. Il nous fallait cependant le mentionner, car avec la contrainte externe que nous avons signalée plus haut, il concourt à déterminer les composantes systémiques qui expliquent (en partie tout au moins) l'écart ethnique que nous avons documenté. Phénomène important aussi parce que généralisable depuis le débat autour de la part aux places jusqu'au cœur de la société bas-canadienne elle-même.

258 Nous utilisons ici une idée développée par G. J. Stigler ("The Economics of Information", Journal of Political Economy, 69, juin 1961) et reprise par J.-L. Migué '"Le Nationalisme, l'unité nationale et la théorie économique de l'information", Revue canadienne d'Economique, 3, mai 1970), pour analyser certains phénomènes de la structure occupationnelle dans l'administration fédérale canadienne des dernières années. Nous ne retenons ici que les bases économiques de l'argumentation, sans tenter de rejoindre soit l'hypothèse précise de Migué, soit sa vérification empirique.

$259 \mathrm{~T}$. Denton, op. cit. 
On sent ce malaise jusque dans la correspondance des privilégiés francophones du temps. Même s'ils fonctionnent à l'intérieur du système, ils n'en perçoivent pas moins le poids aliénant d'un réseau dont ils ne font pas vraiment partie. L.-R.-C. de Léry, qui appartient à une famille déjà privilégiée, esquisse un mouvement d'impatience devant les échecs successifs de son père à lui trouver une place. ${ }^{260}$ Plus intéressant encore est cet aveu de l'honorable M.-A. Panet, l'un des favoris du régime qui a tenté toute sa vie d'étendre le cercle du patronage aux Canadiens français :
... votre lettre d'aujourd'hui m'a fait une nouvelle faveur en m'exprimant la confiance que vous mettez en mon zèle à servir nos infortunés compatriotes Canadiens. Depuis long- temps je les considère très éloignés des faveurs durables, quoique méritées, et craignais qu'il ne fussent comme ils sont aujourd'hui congédiés même du service militaire en leur pays. ${ }^{261}$
Quoique depuis plus de vingt ans j'aye fait tous mes efforts pour notre meilleure Constitution et le bien-être général, et que je croye y avoir réussi à quelqu'égard, néanmoins je n'ai rien acquis en mon particulier, et ne puis donner aucune place convenable à monsieur Montizambert, Enseigne réformé, que vous me recommandez. 262

D'autre part, les conflits concrets (voir section IV) trahissent des phénomènes de fausse conscience fort intéressants : les Canadiens en appellent d'une neutralité et d'intentions bienveillantes (largement imaginaires et parfois contraires à la réalité) de la métropole anglaise pour tarauder les Britanniques coloniaux, sur qui ils rejettent toute la responsabilité de leurs maux.

On pourrait épiloguer sur ces facteurs qui fondent l'insécurité et l'anomie diffuse caractéristiques de la société bascanadienne au tournant du XIX $\mathrm{X}^{\mathrm{e}}$ siècle. Les sociologues ont exposé comment les malaises sociaux de ce type créent un climat

260 “... je m'attendais aux refus de tous les côtés, ils ne m'ont pas surpris, ce sera un troisième sans doute que nous préparons du côté d'Halifax [auprès du duc de Kent] ... [D'ailleurs] je ne crois pas être assez riche pour me soutenir dans le corps du prince... je ne serai jamais en état d'acheter une compagnie... enfin j'ai 33 ans, il est un peu tard [pour] se mettre à la queue d'un régiment..." (L.-R.-C. de Léry à son père, Vaudreuil, $1^{\mathrm{er}}$ janvier 1796, APQ, gr. coll., papiers Chaussegros de Léry, b. 10: s.p.). en 1802 .

261 Il s'agit du licenciement du Corps de volontaires royaux canadiens,

262 L'honorable M.-A. Panet à L. de Salaberry, Québec, 31 juillet 1802, $\mathrm{APQ}$, gr. coll., papiers Salaberry (copies), b. 1: s.p. 
favorable à l'émergence de mouvements "charismatiques". Nous ne poursuivrons pas dans cette voie pour le moment. Nous voulons seulement souligner la valeur exemplaire de la liste civile et la possibilité de repérer, dans l'analyse de cette institution, certains éléments d'explication qui rejoignent la société globale.

Le patronage que nous avons défini un peu comme un "phénomène social total" au sens de Mauss, mérite donc bien plus d'attention qu'on ne lui en a consacrée. Plus qu'un indicateur social ordinaire, la vie concrète qui entoure cette institution est le microcosme de la vie mouvementée de la société qu'il habite. C'est là atteindre peut-être cette "histoire charnelle des institutions" dont parle souvent Albert Faucher.

\section{Conclusion}

La liste civile est un matériau riche pour mordre dans l'histoire globale à partir d'un problème d'économie publique. Le contrepoint dialectique du politique, de l'économique, du social, du mental et de l'ethnique s'y manifeste d'autant plus clairement qu'il s'agit d'une période de transformation et de turbulence. La manière dont l'Etat réagit aux pressions des différents groupes et redistribue les sommes perçues pour répondre à des besoins privés et collectifs qu'il définit selon des biais repérables, affecte l'équilibre entre les groupes ethniques, les classes sociales, les régions, les partis politiques et leurs stratégies. La "part aux places", fixée pour chaque groupe par l'Etat préjugé et influençable, pèse sur toute la gamme des conflits qui crispent le Bas-Canada, entre 1792 et 1812 : depuis les frictions individuelles, familiales et sociales jusqu'à la nation et au système colonial. Aussi avons-nous pu affirmer que la liste civile illustre, reproduit en petit, la situation globale dans la colonie.

Nous avons d'abord deux groupes ethniques. Si chacun existait seul, il aurait à faire face à des problèmes politiques, économiques, sociaux et culturels propres - problèmes compliqués encore par le statut colonial et le contexte nord-américain. On assisterait sans doute - on assiste même à certains égards - ${ }^{263}$ au conflit classique, dans les colonies, entre d'une part, les représentants coloniaux élus à l'Assemblée (des fermiers,

263 Dans le cas de la seule société canadienne-française, l'élite professionnelle et petite-bourgeoise tente en effet de déposter l'ancienne élite seigneuriale sur le plan social et, à un moindre degré, politique (les seigneurs ne contrôlent pas vraiment l'Etat, même s'ils bénéficient de certaines faveurs). 
petits bourgeois, professionnels et marchands) et d'autre part, l'oligarchie des hauts fonctionnaires métropolitains et une caste d'alliés locaux - le "Family Compact" du Haut-Canada.

La situation n'est pas si simple. Il arrive que deux sociétés ethniquement différenciées se bâtissent sur le même territoire, chacune avec ses ambitions collectives et sa vision du monde. Leur affrontement et leur lutte pour le pouvoir gauchissent gravement les conflits "normaux" auxquels il faudrait s'attendre. La société britannique (colons, bourgeois, aristocrates) fait bloc autour de l'Exécutif, le pouvoir et le patronage, et s'identifie aux valeurs britanniques, commerciales et protestantes. L'autre, la canadienne-française - hormis pour l'ancienne élite seigneuriale discréditée —, se groupe également en un front commun, mais à l'Assemblée, et représente surtout l'agriculture, les professions libérales et le catholicisme. L'identification très poussée (mais simplificatrice) entre, d'un côté, les Britanniques et le statut colonial, le commerce, les places, les faveurs, done la richesse et le pouvoir, et de l'autre, les Canadiens et les éléments défavorisés, populaires, coloniaux revendicateurs (sur les plans politique et social), braque ces deux blocs l'un contre l'autre.

Les Canadiens français entendent utiliser le seul instrument en leurs mains, l'Assemblée, pour maçonner une société globale conforme à leur "esprit". ${ }^{264}$ Les Britanniques veulent en faire autant. La lutte des premiers pour la responsabilité ministérielle, la nomination d'un agent en Angleterre et le contrôle des subsides (donc du patronage), se situe à ce niveau global, c'est-àdire à un niveau qui assume et relie tous les paliers d'intérêt et de conflit (individuel, social, national). La confiner au niveau purement politique ou à la crise d'un groupe social particulier, c'est la tronquer. Craig l'a bien compris. ${ }^{265}$

264 C'est-à-dire à leurs intérêts, leurs traditions, leur vision du monde, leur rêve national.

265 Et l'on comprend alors la position inconfortable des quelques Canadiens à places, issus pour la moitié de l'oligarchie seigneuriale. Celle-ci est écartelée entre le désir de renforcer ses privilèges menacés, ses intérêts de classe, et celui, non moins fort, de construire une "nation canadienne" - ce sont les nobles qui utilisent l'expression les premiers, bien qu'ils la conçoivent différemment, bien sûr, des "démocrates" - et de résister à la politique d'assimilation que favorisent les administrateurs coloniaux. Cette ambivalence des aristocrates à places se traduit de bien des façons. Les Britanniques préparent leurs plans assimilateurs entre amis "sûrs" (v.g. en 1801, voir DC II, 268 sqq.). D'autre part, en 1805, l'évêque anglican Mountain accuse les seigneurs canadiens, au Conseil, d'obstruer les mesures en vue de l'érection des paroisses anglicanes ( $\mathrm{J}$. Mountain à Camden, Québec, 24 octobre 1804, APC, M.G. 11, Q. 96: 171-177.). 
Partant d'une morphologie des dépenses publiques, nous nous sommes arrêtés moins sur la mécanique de l'allocation des ressources dans le secteur public que sur les processus sous-jacents à une économie de l'échange tronqué, cet échange apparemment unidirectionnel que constitue le don. ${ }^{266}$ Nous avons cherché à saisir, derrière le patronage, le double flux qui forme l'échange. Ce qui nous a conduits à ausculter les motivations des parties en présence et à discerner, derrière les incidences différenciées du patronage, l'émergence d'un mode de vie politique qui va caractériser les sociétés démocratiques. ${ }^{267}$ Pour nous, la liste civile n'est pas un moment pathologique dans l'évolution d'une société; elle s'avère une institution dont l'apparition, l'évolution et la disparition devront être reconnues à l'aide d'une théorie du changement institutionnel. Celle-ci manque encore dans l'arsenal de l'économie historique. ${ }^{268}$

Faute d'avoir un couplage aussi fort entre l'institution-liste civile et la société globale, nous avons quand même voulu donner un coup de sonde depuis la première vers la seconde. Nous avons rejoint, en partie du moins, la polyvalence des conflits dans l'univers bas-canadien et, au-delà de la spécificité canadienne, certaines caractéristiques plus générales (le biais colonial, le problème des réseaux informationnels). Il faut espérer que cette étude incitera à des analyses parallèles de l'ensemble des institutions bas-canadiennes au tournant $d u X X^{e}$ siècle. C'est cette porte que nous avons voulu entr'ouvrir pour le moment.

\author{
Gilles Paquet \\ (Carleton University) \\ Jean-Pierre Wallot \\ (Université de Toronto)
}

266 Pour l'amorce d'une analyse de l'économie du don, voir F. Perroux, Economie et société (Paris, 1960); B. de Jouvenel, "The Political Economy of Gratuity", The Virginia Quarterly Review, 35, automne 1959.

267 A. Downs, An Economic Theory of Democracy (New-York, 1957). 268 Les premiers efforts sont amorcés cependant (L. Davis \& D. North, "Institutional Change and American Economic Growth: A First Step Towards a Theory of Institutional Innovation", Journal of Economic History, $30, n^{\circ} 1$ (mars 1970) : 131-149. 


\section{APPENDICE A}

\section{LES DONNÉES}

Toutes les données qui ont servi aux analyses quantitatives proviennent des comptes publics du Bas-Canada. Ceux-ci paraissent chaque année, dans les Journaux de la Chambre d'Assemblée avec un décalage d'une année : v.g. le Journal de 1800 reproduit les chiffres pour 1799 et ainsi de suite. Les comptes publics détaillent tous les revenus de l'Etat selon leur origine (revenus des terres et propriétés de la Couronne, droits imposés par des lois impériales et coloniales), en les confrontant aux dépenses et aux commissions diverses s'il y a lieu. La liste civile comprend presque toutes les dépenses : salaires des fonctionnaires, commissions des percepteurs, dépenses pour l'application des diverses lois (v.g. construction des prisons de Montréal et de Québec après 1805), quelques contrats (surtout d'imprimerie) et loyers, les dépenses encourues par les fonctionnaires dans l'exercice de leurs fonctions, etc. De là le danger de duplication, puisque la liste civile répète certains salaires, commissions ou dépenses qui sont enregistrés ailleurs vis-à-vis les revenus de telle ou telle loi. Seuls quelques emplois spécifiques pourvus par deux lois spéciales de l'Assemblée (service législatif, pilotage sur le Saint-Laurent) ne sont pas reproduits dans la liste civile, de sorte que nous avons dû en tenir compte et les ajouter à nos compilations (voir section $\mathrm{V}, 1$, pour des précisions supplémentaires).

Nous avons déjà expliqué certaines difficultés que présente l'utilisation de ces sources. Parfois, la liste civile identifie clairement un fonctionnaire et son poste (v.g. "l'hon. G.-E. Taschereau, grand voyer du district de Québec, $£ 150.0 .0$ st."). A d'autres moments, l'identification est difficile (il n'y a pas de prénom ou pas de nom du tout, v.g. "commis") ou les précisions manquent quant à la nature du paiement effectué par l'Etat : "pour services spéciaux", "allouances" "allouances et honoraires", "pour dépenses" en vertu de telle loi, etc. Dans les pensions, en particulier, on trouve les deux extrêmes: depuis quelqu'un qui reçoit tant pour une infirmité qui a résulté d'une blessure subie lors du siège de Québec, en 1775-1776, jusqu'à une "dame Cabanac" en "détresse". Mais dans le cas des salaires, la liste civile reproduit en général les initiales, le nom propre, l'occupation, souvent la région et la rémunération du fonctionnaire. Ce qui nous a permis de transcrire une partie de ces informations en une forme purement numérique. Par exemple, "l'hon. G.-E. Taschereau, grand voyer du district de Québec, $£ 150.5 .11$ st." devient "21151500511", où

$$
\begin{aligned}
2= & \text { Canadien français; } \\
1= & \text { région de Québec; } \\
1= & \text { livre sterling; } \\
5= & \text { catégorie } 5 \text { (de } £ 100 \text { à } £ 200 \text { st.), information qui n'est pas } \\
& \text { nécessaire cependant, puisque l'ordinateur peut reconstituer } \\
& \text { toute catégorie de notre choix automatiquement; } \\
150= & £ 150 ;
\end{aligned}
$$


$05=5$ shillings $(20$ shillings à la livre $)$;

$11=11$ deniers ( 12 deniers ou pences au shilling).

Nous avons expliqué ailleurs pourquoi nous n'avons pu codifier l'origine sociale (voir $\mathrm{V}, 2$ ).

Voici les renvois précis pour nos données: 1794, JCABC (1795), 83-102; 1795, JCABC (1796), 143-144, 147-166, 169-172; 1796, JCABC (1797), 149-150, 153-166, 169-170; 1797, JCABC (1798), 105-106, 111112, 124-132; 1798, JCABC (1799), 72-73, 78-79, 89-95; 1799, JCABC (1800), 188-189, 194-195, 206-213; 1800, JCABC (1801), 254-255, 260-261, 274-283; 1801, JCABC (1802), 168-169, 174-175, 178-195; 1802, JCABC (1803), 72-73, 80-81, 86-105; 1803, JCABC (1804), 124125, 142-143, 168-177; 1804, JCABC (1805), 324-325, 342-343, 354-363; $1805, J C A B C$ (1806), 178-179, 198-199, 212-223; 1806, JCABC (1807), 224-225, 246-247, 260-271; 1807, JCABC (1808), 356-357, 378-379, 393405 ; 1808, JCABC (1809), app. 8, 16 et $17 ; 1809, J C A B C$ (1811), app. A, 8, 18 et 19; 1810, ibid., app. B, 8, 20 et 21; 1811, JCABC (1812), app. 8,18 et $19 ; 1812, J C A B C(1813)$, app. E, 6, 17 et 18 . Nous avons précisé les autres sources dans nos notes.

\section{APPENDICE B}

\section{NOTE SUR LES TECHNIQUES STATISTIQUES}

Nous avons utilisé dans notre mémoire un certain nombre de mesures et de techniques statistiques. Bien que ces concepts et ces outils soient connus et d'usage courant chez les spécialistes de sciences humaines, ils ne font pas encore partie de l'univers familier de l'historien. Il ne saurait être question de faire ici un cours de statistiques appliquées; mais il nous a paru utile de tracer à gros traits une esquisse du cadre général qui soustend les exercices statistiques que nous avons présentés plus haut et d'attirer l'attention sur certaines techniques plus spéciales auxquelles nous avons eu recours, bien qu'elles ne soient pas encore partie du matériel présenté dans les manuels élémentaires.

a) Une variable est une caractéristique qui change d'une chose ou d'un individu à l'autre à l'intérieur d'une population. L'on peut distinguer deux sortes de variables : les variables numériques et les variables attributs. L'âge ou le niveau du revenu de chaque employé civil du Bas-Canada sont des exemples de variables numériques, alors que l'origine ethnique ou la localisation géographique de ces employés sont des attributs. Une statistique s'avère un repère synthétique d'un phénomène mesuré par une variable. Nous avons utilisé dans la section $\mathrm{V}$ des moyennes arithmétiques annuelles pour représenter les niveaux de salaire et de pension. Il s'agit d'une mesure de localisation du phénomène. La moyenne arithmétique repère en quelque sorte le centre 
de gravité de la masse informationnelle définie par la variable. Elle constitue une condensation de cette masse en un seul nombre qui en établit la localisation. On aurait pu définir plus précisément la masse en question, en en mesurant la distribution, la variation, la symétrie, etc., mais nous nous sommes contentés ici de la seule mesure de localisation. Il est important de noter que la moyenne arithmétique est une mesure qui s'applique également aux variables numériques et aux variables attributs. Cependant, dans ce dernier cas, il faut avoir recours à un procédé ingénieux pour $\mathrm{y}$ arriver. Ce procédé consiste à donner la valeur 1 à la chose ou la personne qui possède l'attribut qui nous intéresse et une valeur 0 à celle qui ne le possède pas. Les variables qui prennent seulement les valeurs 0 et 1 sont appelées variables fictives. Ainsi, si nous utilisons la classification noir-blanc et que nous attribuons la valeur 1 à chaque individu de la population qui est noir, et la valeur 0 aux autres, il devient possible de faire la somme de ces valeurs et de diviser le tout par le nombre total des observations. La moyenne arithmétique d'une variable fictive est de fait une proportion. Dans le cas qui nous occupe, ce sera la proportion des noirs dans la population.

b) L'une des caractéristiques importantes de la moyenne arithmétique est que la somme des carrés des écarts entre chaque valeur de la variable pour chaque membre de la population et la moyenne est plus petite que la somme des carrés des écarts entre chaque valeur de la variable pour chaque membre de la population et tout autre nombre différent de la moyenne. C'est là une caractéristique clé qui nous a servi dans nos analyses. En effet, nous ne nous sommes pas contentés de repérer la moyenne arithmétique de certaines variables numériques et de certains attributs. Nous avons poussé l'analyse plus avant dans la section VI. Là, nous avons voulu mesurer l'association entre deux ou plusieurs variables. En effet, nous avons cru que si nous pouvions distinguer un certain nombre de sous-catégories à l'intérieur de la population qui nous intéresse, il pourrait être possible d'observer des covariations entre une variable $Y$, disons, et la caractéristique $X$ que nous retenons comme principe de définition des sous-catégories. C'est ainsi qu'au lieu de nous satisfaire d'une moyenne de salaire pour la période 1794-1812, nous avons voulu découper la masse des informations sur les salaires selon les années, selon les groupes ethniques et selon les régions, pour mesurer des moyennes arithmétiques correspondant à ces sous-portions de la population. En fait, l'analyse de régression que nous avons utilisée est le point limite d'un procédé de ce genre. L'analyse de régression consiste à identifier sur un plan YX (quand on examine la relation entre deux variables seulement) ou dans un espace multidimensionnel $\mathbf{Y X}_{1} \mathrm{X}_{2} \mathrm{X}_{3}$ etc. (quand il s'agit de la relation qui soude plusieurs variables) la ligne, le plan ou l'hyperplan qui est le plus représentatif des observations enregistrées. Pour fixer les idées, nous pouvons dire que cette ligne dans un espace à deux dimensions YX est le lieu des moyennes conditionnelles, i.e. le lieu des moyennes arithmétiques de $\mathrm{Y}$ pour certaines valeurs précisées 
à partir de $\mathrm{X}$. On peut mieux saisir le sens de cette opération à l'aide des deux graphiques suivants qui représentent l'opération intermédiaire et l'opération limite de la production de ces moyennes conditionnelles. L'un définit les moyennes conditionnelles pour un nombre restreint de catégories spécifiées par $X$ alors que l'autre définit les moyennes conditionnelles pour chacune des valeurs possibles de $\mathrm{X}$.
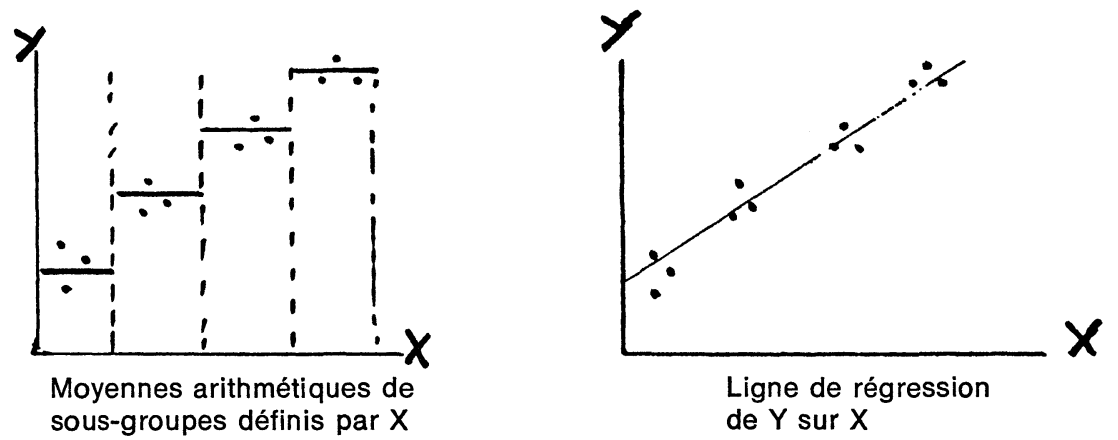

La ligne de régression trace le lieu de toutes les moyennes conditionnelles de $Y$ pour toutes les valeurs de $X$. On peut définir précisément la localisation de cette ligne en en spécifiant la forme et les coefficients. Dans ce mémoire, nous nous en sommes tenus à la forme linéaire. Mais nous avons de fait défini des hyperplans dans des espaces multidimensionnels puisque nos régressions impliquaient une multiplicité de variables indépendantes (i.e. $X_{1}, X_{2}, X_{3}$, etc.). Quant aux coefficients qui soudent les variables, on peut les estimer à partir de cette propriété de la moyenne dont il a été question auparavant. En effet, si la moyenne arithmétique est le nombre qui minimise la somme des carrés des écarts, nous pourrons repérer la ligne de régression (i.e. la ligne des moyennes conditionnelles) en ajustant dans le plan ou l'hyperplan une ligne qui soit celle des moindres écarts, i.e. telle que la somme des carrés des écarts entre la ligne et tous les points observés soit la plus petite possible. Dans le cas du plan YX, une telle ligne sera définie précisément par le coefficient angulaire et la pente. Dans le cas d'un espace YXZ, le plan sera défini par le coefficient angulaire, la pente dans la direction YX et la pente dans la direction YZ.

c) Une fois estimée la ligne de régression, il nous reste à évaluer le degré de confiance que nous pourrons avoir dans ces moyennes conditionnelles qu'elle nous permet de produire. Ainsi, puisqu'à partir de toute valeur de $\mathrm{X}$, nous pouvons maintenant engendrer via la ligne de régression une moyenne arithmétique de $\mathrm{Y}$ valable pour cette valeur de $\mathrm{X}$, nous ne nous contenterons plus d'une moyenne arithmétique générale pour $Y$. Notre connaissance de $X$ nous permettra de serrer de bien plus près la valeur de $\mathrm{Y}$ qui est pertinente. C'est ce 
processus qui permet d'utiliser notre connaissance d'une variable $\mathbf{X}$ pour réduire notre incertitude à propos d'une autre variable Y. Reste à savoir si les prédictions faites à partir de cette ligne de régression sont fiables. Pour ce faire, nous avons utilisé deux séries de tests : le coefficient de détermination qui jauge la crédibilité de la ligne de régression in toto et des tests du type $t$ pour jauger plus particulièrement la crédibilité des coefficients de pente. Le sens de ces deux types de tests est fort simple. Dans le cas du coefficient de détermination $\left(R^{2}\right)$ que nous avons attaché à chaque équation, il s'agit, ainsi que nous l'avons montré à la note 176 , d'une mesure de covariation entre la variable dépendante et le faisceau des variables indépendantes. Plus précisément, $\mathbf{R}^{2}$ mesure le pourcentage de la variation de $\mathrm{Y}$ imputable à une variation dans $X$ (ou dans le faisceau de toutes les variables indépendantes). Il ne s'agit pas ici d'une relation causale, mais d'une imputation strictement statistique. Comme en l'absence de toute connaissance de $X$, le seul recours serait de mesurer la moyenne générale de $Y(\bar{Y})$, le degré d'imprécision de cette mesure serait spécifié par l'écart entre le point observé et cette moyenne générale $(\bar{Y})$. Cependant si nous connaissons $X$, nous pouvons faire mieux et définir, pour ce point précis qui nous intéresse, une moyenne conditionnelle. Cette moyenne conditionnelle, on l'obtient via la ligne de régression. Le problème est de savoir si la moyenne conditionnelle est un meilleur estimé de la réalité observée que la moyenne générale. Si tel est le cas, on enregistrera un écart plus petit entre le point observé et la moyenne conditionnelle qu'entre le point observé et la moyenne générale. Cette réduction de l'écart sera ainsi imputable à la connaissance de $X$. $R^{2}$ mesure la proportion de l'écart entre le point observé et la moyenne générale $(\bar{Y})$ qui est éliminée par l'injection d'une connaissance de $\mathrm{X}$. $\mathrm{Si}$ nous simplifions, nous pouvons représenter $\mathrm{R}^{2}$ graphiquement comme suit :

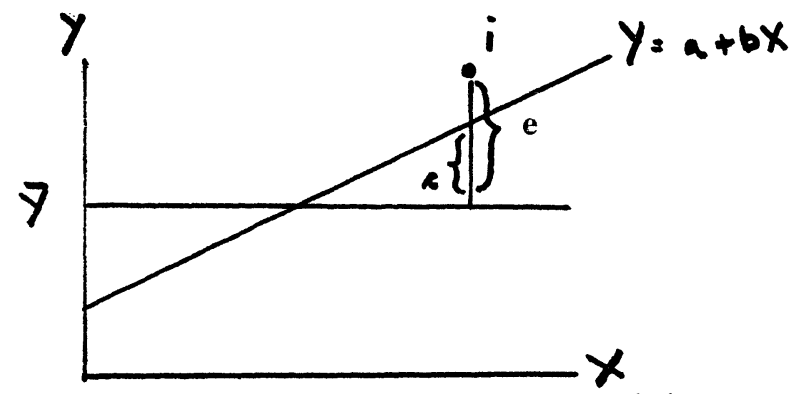

L'écart entre le point observé $\mathrm{i}$ et la moyenne générale est dénoté(e), et l'écart entre le point observé et la moyenne conditionnelle égale $(\mathrm{e}-\mathrm{c})$. $\mathbf{R}^{2}$ mesure la proportion $\mathrm{c} / \mathrm{e}$ à toutes fins utiles. Afin de pouvoir agréger toutes sortes de valeurs positives et négatives (pour les points qui seraient sous la ligne des moyennes), ce rapport est effectué en termes de carrés des écarts. 
Pour ce qui est du test des coefficients de pente, il s'agit d'un test nommé t qui, ainsi que nous l'indiquions à la note 177 , compare la valeur du coefficient de pente avec une mesure de sa variabilité. Ainsi que nous l'avons vu au graphique 3 , des modèles différents appliqués à des données identiques peuvent fort bien produire des lignes de régressions différentes, i.e. à coefficients différents. Or même si nous nous en tenons à un seul modèle, il est possible d'imaginer plusieurs pentes possibles que l'on pourrait ajuster à une série d'observations données. Plus la variation des points observés de chaque côté de la ligne de régression est ample, et plus le domaine sur lequel s'étendent les valeurs de $X$ est étroit, plus la variation possible du coefficient de pente est grande. Daniel Suits a utilisé une métaphore fort heureuse pour approximer ce problème; il parle de la ligne de régression comme d'un bâtonnet inséré dans un tuyau. Si le tuyau (qui définit le lieu des points observés) est long et étroit, la variabilité de la pente du bâtonnet est limitée; mais si le tuyau est court et gros, le bâtonnet peut prendre toute une gamme de pentes différentes. Le test $t$ consiste à jauger si le coefficient de pente est assez grand relativement à cette marge d'erreur définie par l'erreur-standard pour qu'on puisse dire que le coefficient observé est différent de 0 . L'importance de ce test, c'est qu'il permet de repérer à l'intérieur d'une équation complexe qui en général est significative, quels sont les éléments qui peuvent fort bien ne pas l'être. Comme la valeur critique du rapport $\mathrm{t}$ change avec le nombre des observations et avec le nombre de variables indépendantes, nous renvoyons aux tables spécialisées pour la mesure exacte du degré de signification. En général cependant, étant donné l'ordre de grandeur de notre population, la règle simple suggérée à la note 177 est fort acceptable.

d) Notre mémoire a utilisé comme variables dépendantes et des variables numériques et des variables attributs. Dans le cas du patronage de rétribution, la variable dépendante est un salaire ou une pension, alors que dans le cas du patronage de répartition, la variable dépendante est une ethnie ou une région. Alors que les variables numériques sont généralement utilisées, on doit dire qu'il est plus rare de voir des variables attributs dans ce type d'analyse. Dans ce dernier cas, l'attribut revêt seulement les valeurs 0 et 1 , ce qui veut dire que la variable $\mathbf{Y}$ dans notre analyse est restreinte à ce domaine. L'estimation des moyennes dans le cas de variables dichotomiques de ce genre revient à une mesure de proportions. Ainsi, la même analyse de régression va consister à engendrer une ligne des proportions conditionnelles qui sera comparée à la proportion générale. C'est par une extension de la notion de proportion que nous en arrivons à parler de probabilité dans notre mémoire. Le passage est légitime puisque la probabilité se définit comme la fréquence d'un phénomène, i.e. le nombre de fois où le phénomène se produit par rapport au nombre total des observations. A ce propos, nous référons le lecteur à l'excellent ouvrage de G. H. Orcutt, M. Greenberger, J. Korbel et A. M. Rivlin, Microanalysis of Socio-Economic Systems; a Simulation Study, Harper, New-York, 1961. 
e) Pour ceux qui voudraient un exposé plus complet des détails de ces calculs et des postulats qui sous-tendent ces analyses, ils pourront se reporter à l'un ou l'autre des manuels cités dans les notes 176, 177 et 178, ainsi qu'à l'excellent manuel de E. J. Kane, Economic Statistics and Econometrics; An Introduction to Quantitative Economics, NewYork, 1968.

G. P.

J.-P. W. 